\title{
Supersymmetric D3/D5 for massive defects on curved space
}

\author{
Brandon Robinson $^{a}$ and Christoph F. Uhlemann ${ }^{a, b}$ \\ ${ }^{a}$ Department of Physics, University of Washington, \\ Seattle, WA 98195-1560, U.S.A. \\ ${ }^{b}$ Mani L. Bhaumik Institute for Theoretical Physics, Department of Physics and Astronomy, \\ University of California, \\ Los Angeles, CA 90095, U.S.A. \\ E-mail: robinb22@uw.edu, uhlemann@physics.ucla.edu
}

ABStract: We construct the holographic dual for $\mathcal{N}=4 \mathrm{SYM}_{\text {on }} \mathrm{S}^{4}$ and $\mathrm{AdS}_{4}$ coupled to massive $\mathcal{N}=2$ supersymmetric quenched flavor fields on a codimension-1 defect, which is $\mathrm{S}^{3}$ and $\mathrm{AdS}_{3}$, respectively. The holographic description is in terms of a D3/probe D5 brane system. We set up and reduce the BPS equations for D5-brane embeddings with arbitrary supersymmetric deformations and partly solve them at the non-linear level. The remaining equations are solved explicitly in a small-mass expansion. We compute the contribution of the defect fields to the partition function on $\mathrm{S}^{4}$ and compare to a field theory computation using supersymmetric localization, for which we set up the matrix model. Both computations agree, lending strong support to holographic probe brane constructions using D3/D5 configurations in general.

KeYwords: AdS-CFT Correspondence, D-branes, Matrix Models

ArXiv EPrint: 1709.08650 


\section{Contents}

1 Introduction and summary 1

2 Reduced BPS equations for $\mathrm{AdS}_{3}$ defects $\quad 7$

2.1 Background geometry and embedding ansatz 8

$2.2 \kappa$-symmetry generalities $\quad 9$

$\begin{array}{lll}2.3 & \text { Preserved supersymmetries } & 10\end{array}$

2.4 Non-linear $\kappa$-symmetry 15

2.5 The BPS equations 16

$\begin{array}{ll}2.6 & \text { Solving for the gauge field } \\ & 18\end{array}$

$\begin{array}{lll}2.7 & \text { The remaining equations } & 19\end{array}$

3 Perturbative solutions for $\mathrm{AdS}_{3}$ defects 20

$4 \mathrm{~S}^{3}$ defects and $\mathrm{S}^{4}$ partition function from holography $\quad 25$

$\begin{array}{lll}4.1 & \text { Continuing perturbative solutions } & 26\end{array}$

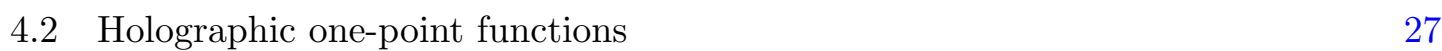

4.3 Sphere partition function 30

$5 \quad \mathrm{~S}^{4}$ partition function from supersymmetric localization $\quad 31$

5.1 The matrix model 31

$\begin{array}{lll}5.2 & \mathrm{~S}^{4} \text { partition function } & 32\end{array}$

A Details on non-linear $\kappa$-symmetry $\quad 34$

\section{Introduction and summary}

Quantum field theory on curved space has recently seen a revival of interest for purely formal reasons: by reducing the path integral to a smaller subset of field configurations, supersymmetric localization allows one to exactly and non-perturbatively compute BPS observables such as the partition function or supersymmetric Wilson loops [1]. To guarantee well-defined answers, compact Euclidean spaces are preferable, and to realize a maximal amount of symmetry spheres are a natural first choice. As a simple generalization to backgrounds with a lesser amount of symmetry, continuous deformations such as squashed spheres have been studied [2].

In particular in connection with the conjectured AdS/CFT dualities, the prospect of obtaining exact results at strong coupling offers the chance to confront the holographic dualities with decisive tests. With full non-perturbative results from purely field-theoretic calculations in hand, one can compare to a holographic computation of the same quantity 
and test whether the two pictures agree. The tests performed to date include dualities for field theories in various dimensions [3-5], dualities involving probe brane constructions [6] and many more. While agreement of the two calculations is still not a formal proof for any of the dualities, these tests against localization calculations provide a powerful tool to potentially disprove the dualities, and the agreement on highly non-trivial observables provides strong circumstantial evidence for the validity of the dualities.

In interesting recent developments, first steps were taken to study localization on antide Sitter spacetimes [7, 8]. In [7], calculations on $\mathrm{AdS}_{2} \times \mathrm{S}^{1}$ were performed and checked, by relating the $\mathrm{AdS}_{2} \times \mathrm{S}^{1}$ geometries to covering spaces of $\mathrm{S}^{3}$. Studying field theories on AdS is interesting for a number of reasons, not the least of which is that it introduces a boundary and dependence on boundary conditions in a maximally symmetric way. The study of boundary conditions on AdS has been pioneered by Breitenlohner and Freedman $[9,10]$, and their results are ubiquitous in AdS/CFT. Their studies focus on free or perturbatively interacting fields, and to study the impact of boundary conditions on AdS for interacting field theories the AdS/CFT correspondences once again have been of great utility [11]. Coming back to testing the dualities using localization, the main obstacles that need to be overcome to extend the tests done so far to include AdS spacetimes are on the field theory side - on the holographic side the calculations for field theories on AdS are closely parallel to the ones for spheres. In fact, certain extra subtleties, e.g. with Euclidean supersymmetry, can even be avoided on AdS. Holographic results can thus provide useful guidance for the field theory developments and facilitate consistency checks. A study involving the impact of boundary conditions in a different context using localization was performed in [12].

In both cases, for field theories on $\mathrm{AdS}_{d}$ and on $\mathrm{S}^{d}$, constructing the holographic dual amounts to finding asymptotically- $A d S_{d+1}$ supergravity solutions where the metric at the conformal boundary of $\mathrm{AdS}_{d+1}$ is $\mathrm{AdS}_{d}$ or $\mathrm{S}^{d}$, respectively. For a conformally invariant theory the supergravity solution can still be globally $\mathrm{AdS}_{d+1}$, and the boundary metrics for $\mathrm{AdS}_{d}$ and $\mathrm{S}^{d}$ can be realized by a mere change of coordinates. This reflects that $\mathrm{AdS}_{d}$ and $\mathrm{S}^{d}$ are representatives of the same (conformally flat) conformal structure as flat space. However, to obtain physically meaningful results, it is often necessary to study deformations of the CFT that introduce a scale. An example is the sphere partition function in even dimensions. It is a simple example of a computable quantity in localization and naturally suggests itself for comparison to holographic results. But it can be shifted by constants due to the presence of finite counterterms and is therefore not independent of the choice of renormalization scheme. For a more detailed discussion see e.g. [13]. This scheme dependence can be cured by studying supersymmetric mass deformations of the CFT of interest, such that the partition function becomes a function of the dimensionless product of mass parameter and radius of the sphere. Once conformal invariance is broken, it does make a difference whether the theory is considered on flat space, on $\mathrm{AdS}_{d}$ or on $\mathrm{S}^{d}$, and finding the holographic duals for the mass deformations becomes more involved. But the free energy then contains scheme-independent and non-trivial information and allows for non-trivial tests. This is the strategy followed for the tests on $\mathrm{S}^{4}$ e.g. in [3] and [6].

The results of [6] showed that the field theory calculation of the free energy for $\mathrm{SU}\left(N_{c}\right)$ $\mathcal{N}=4 \mathrm{SYM}$ coupled to $N_{f} \ll N_{c}$ massive hypermultiplets in the fundamental repre- 
sentation, at strong coupling on $\mathrm{S}^{4}$, agrees with the holographic calculation of the same quantity. The holographic computation starts out from the $\mathrm{AdS}_{5} \times \mathrm{S}^{5}$ solution to type IIB supergravity, into which $N_{f}$ probe D7 branes describing the flavor degrees of freedom are embedded. For $N_{f} \ll N_{c}$ the backreaction of the D7 branes can be neglected, which considerably simplifies the computation: since the mass deformation only affects the flavor fields, the background $\mathcal{N}=4 \mathrm{SYM}$ theory can be realized on $\mathrm{S}^{4}$ by a mere change of coordinates on $\mathrm{AdS}_{5}$. Only the $\mathrm{D} 7$ branes are affected by the mass deformation and realizing the holographic setup amounts to finding the appropriate embedding into $\operatorname{AdS}_{5} \times \mathrm{S}^{5}$. We found those embeddings for both, $\mathrm{AdS}_{4}$ and $\mathrm{S}^{4}$ in [14]. The result for $\mathrm{S}^{4}$ allowed us to test the holographic calculation against field theory results, where the probe limit $N_{f} \ll N_{c}$ indeed simplified the calculations drastically as well. The $\mathrm{AdS}_{4}$ case led to interesting phenomenology which we studied in some detail in [14]. The choice of boundary conditions at the conformal boundary of the $\mathrm{AdS}_{4}$ field theory geometry indeed plays a crucial role, and the free energy obtained from those embeddings should provide a good benchmark for field theory calculations.

In this work we will further extend the holographic studies of field theories accessible to localization calculations. We will stay within the realm of probe brane constructions, since they allow to add interesting features to the field theory with only a moderate amount of additional complexity, and consider a quantum field theory with a codimension- 1 defect. More specifically, we will consider the D3/D5 system [15-17], ${ }^{1}$ describing $\operatorname{SU}\left(N_{c}\right) \mathcal{N}=4$ SYM coupled to a number $N_{f} \ll N_{c}$ of fundamental hypermultiplets confined to a threedimensional subspace. We will consider $\mathrm{AdS}_{4}$ and $\mathrm{S}^{4}$ backgrounds for the $\mathcal{N}=4 \mathrm{SYM}$ theory, with defects given by $\mathrm{AdS}_{3}$ and $\mathrm{S}^{3}$, respectively. We will focus on $\mathrm{AdS}_{3}$ defects for the first part and discuss the $\mathrm{S}^{3}$ case afterwards using analytic continuation. The defect breaks part of the isometries of the background as well as the conformal symmetries acting non-trivially in the directions transverse to the defect. But as long as no additional dimensionful parameters are introduced, the theory still preserves a combination of the broken isometries and conformal isometries, which combine with the transformations along the defect to the defect conformal symmetry $\mathrm{SO}(2,3)$. This is the same group for $\mathrm{AdS}_{3}$ defects and flat $\mathbb{R}^{1,2}$ defects, and turns into $\mathrm{SO}(1,4)$ for $\mathrm{S}^{3}$. Correspondingly, already for a flat defect it turns out to be fruitful to think of the "background" $\mathcal{N}=4 \mathrm{SYM}$ as defined on two copies of $\mathrm{AdS}_{4}$ joined at their respective conformal boundaries, which makes the preserved symmetries more manifest. Holographically this again simply amounts to a change of coordinates on $\mathrm{AdS}_{5}$ to realize an $\mathrm{AdS}_{4}$ slicing. The supersymmetric embedding of D5 branes, described by the DBI action

$$
S_{\mathrm{D} 5}=-T_{5} \int_{\Sigma_{6}} d^{6} \xi \sqrt{-\operatorname{det}(g+F)}+T_{5} \int_{\Sigma_{6}} C_{4} \wedge F,
$$

in all cases with preserved defect conformal symmetry wraps an $\mathrm{AdS}_{4}$ subspace of $\mathrm{AdS}_{5}$ and an $S^{2}$ in $S^{5}$. The embedding is illustrated in figure 1 . But as soon as we wish to consider mass deformations or otherwise break conformal symmetry, it makes a difference whether the defect is flat $\mathbb{R}^{1,2}, \mathrm{AdS}_{3}$ or $\mathrm{S}^{3}$. This amounts to the need for different D5-brane

\footnotetext{
${ }^{1} \mathrm{~A}$ large body of work on D3/D5 systems based on integrability can be found in [18-22], a recent study of Wilson lines is in [23] and a discussion of Mellin representations in dCFT can be found in [24].
} 


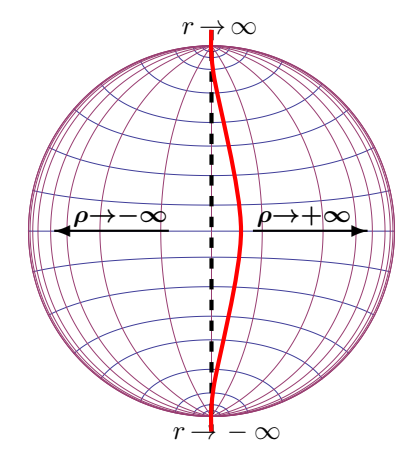

Figure 1. Poincare disc representation of the $\mathrm{AdS}_{4}$ slicing of $\mathrm{AdS}_{5}$ in coordinates (1.2), (1.3). Thin vertical curves are $\mathrm{AdS}_{4}$ slices, while the horizontal curves correspond to slices of constant $r$. The black dashed line shows the $\mathrm{AdS}_{4}$ slice wrapped by the D5 brane for massless flavors, while the thick solid red curve schematically shows an embedding for massive flavors.

embeddings for $\mathrm{AdS}_{3}$ and $\mathrm{S}^{3}$ defects as compared to a flat $\mathbb{R}^{1,2}$ defect (for which massive embeddings have been discussed already in [15]).

The D5 brane embeddings for massive flavors are expected to take the general form illustrated in figure 1, and constructing them explicitly is the task at hand. For the $\mathrm{AdS}_{5} \times \mathrm{S}^{5}$ background geometry created by the D3 branes we choose coordinates such that

$$
g_{\mathrm{AdS}_{5} \times \mathrm{S}^{5}}=d \rho^{2}+\cosh ^{2} \rho g_{\mathrm{AdS}_{4}}+d \theta^{2}+\cos ^{2} \theta g_{\mathrm{S}^{2}}+\sin ^{2} \theta g_{\tilde{\mathrm{S}}^{2}},
$$

with $\rho \in \mathbb{R}$. For the $\mathrm{AdS}_{4}$ metric we choose an $\mathrm{AdS}_{3}$ slicing to realize an $\mathrm{AdS}_{3}$ defect. We will need explicit parametrizations of the $\mathrm{AdS}_{4}$ and $\mathrm{S}^{2}$ metrics and choose

$$
g_{\mathrm{AdS}_{4}}=d r^{2}+\cosh ^{2} r g_{\mathrm{AdS}_{3}}, \quad g_{\mathrm{S}^{2}}=d \beta_{1}^{2}+\sin ^{2} \beta_{1} d \beta_{2}^{2} .
$$

The conformal boundary consists of the two $\mathrm{AdS}_{4}$ geometries obtained as $\rho \rightarrow \pm \infty$, which are joined at their respective conformal boundaries. Moreover, sending $r \rightarrow \pm \infty$ for fixed $\rho$ yields two $\mathrm{AdS}_{3}$ geometries, which are also joined at their conformal boundaries and correspond to a codimension-1 subspace in the boundary geometry. For the D5-brane embedding we choose static gauge with $\left(r, \beta_{1}, \beta_{2}\right)$ and the $\mathrm{AdS}_{3}$ directions as worldvolume coordinates, such that the embedding is characterized by the slipping mode, $\theta$, parametrizing the $S^{2}$ wrapped inside $S^{5}$, and the bending mode, $\rho$, parametrizing the $\mathrm{AdS}_{3}$ slice wrapped in $\mathrm{AdS}_{5}$. The slipping and bending modes are both restricted to be independent of the $\mathrm{AdS}_{3}$ coordinates. The D5-branes intersect the conformal boundary at the two $\mathrm{AdS}_{3}$ geometries obtained as $r \rightarrow \pm \infty$ and describe fundamental fields localized to this subspace of the conformal boundary. The embedding generally preserves the $\mathrm{AdS}_{3}$ isometries, and for identically vanishing $\rho$ wraps an entire $\mathrm{AdS}_{4}$ slice. Once the defect flavors are massive, defect conformal symmetry is broken and the subspace wrapped in $\mathrm{AdS}_{5}$ is not necessarily $\mathrm{AdS}_{4}$ anymore. Moreover, turning on the mass deformation preserves only a $\mathrm{U}(1)$ subgroup of the $\mathrm{SU}(2) \mathrm{R}$-symmetry, and correspondingly the embedding only preserves a $\mathrm{U}(1)$ subgroup of the $\mathrm{SU}(2)$ isometries of the $\mathrm{S}^{2}$ in $\mathrm{S}^{5}$. We can choose the coordinates such that the $\mathrm{U}(1)$ acts by shifting the polar angle $\beta_{2}$, and consequently the worldvolume 
fields are independent of $\beta_{2}$. That means the slipping and bending mode can both depend on the radial coordinate $r$ as well as on the azimuthal angle on the $\mathrm{S}^{2}, \beta_{1}$, and the BPS equations that need to be solved to find supersymmetric embeddings are genuine PDEs. In addition to that, we find that massive supersymmetric embeddings generically require - in addition to non-trivial slipping and bending modes - also flux on the $\mathrm{S}^{2}$, i.e. a nontrivial worldvolume gauge field on the D5 branes. In field theory terms, the requirement for non-trivial fields in addition to the slipping mode sourcing the mass term represents the need for an extra term required to accompany the mass deformation for supersymmetry to be preserved on curved space, as discussed systematically in [25]. Finding supersymmetric embeddings thus amounts to solving non-linear coupled PDEs for three functions, $\rho, \theta$ and $A_{\beta_{2}}$, of two variables, $r$ and $\beta_{1}$.

Setting up the BPS equations for the D5 branes and reducing them to a minimal set of consistent equations constitutes a non-trivial technical part of the analysis, and we solve it in section 2.4. We set up the conditions imposed by $\kappa$ symmetry [26-28] for our embedding ansatz spelled out in section 2.1, and derive the eight resulting constraints on the embeddings. The equations have non-linear coefficient functions and are non-linear in derivative terms as well. Nevertheless, we can isolate an equation that can be solved for the gauge field in terms of the slipping and bending modes. The result is, with $\tanh (r / 2)=$ $\tan (z / 2), x=\cos \beta_{1}$ and a parameter $\lambda$ satisfying $\lambda^{2}=1$,

$$
A_{\beta_{2}}=\lambda \tan z \cosh \rho \sin \theta-x \sinh \rho \cos \theta+\mathcal{A}_{0},
$$

where $\mathcal{A}_{0}$ is a constant. The remaining equations can then be reduced to a minimal set of two equations determining slipping and bending mode. Together with the solution for the gauge field, they imply the entire set of conditions imposed by $\kappa$-symmetry. The remaining two equations are

$$
\begin{aligned}
-\left(1-x^{2}\right) \cosh \rho G_{x}+\sinh \rho \cos \theta F_{z} & =0 \\
\sinh \rho\left(G_{z} F_{x}-G_{x} F_{z}\right)-\cos ^{3} \theta G_{z}-\sec ^{2} z \cosh ^{3} \rho \cos ^{3} \theta & =0 .
\end{aligned}
$$

with $G_{\mathfrak{a}}=\lambda \sinh \rho \sin ^{2} \theta \partial_{\mathfrak{a}}(x \cot \theta)-\partial_{\mathfrak{a}}(\tan z \cosh \rho)$ and $\mathfrak{a}=r, x$. At that level of generality, they encode not only mass deformations, but deformations by any of the supersymmetric operators sourced by combinations of slipping mode, bending mode and gauge field. More specifically, expanding all three fields in harmonics on $\mathrm{S}^{2}$ yields a discrete series of fields on the AdS part of the background geometry. Each of these fields sources a different operator. The remaining equations do not contain square roots, as would be typical for the equations of motion resulting from DBI actions with non-trivial Wess-Zumino terms, but we have not been able to solve them in closed form. The equations can, however, be solved straightforwardly in a perturbative expansion around the massless embedding with no other sources or vacuum expectation values turned on, which we will refer to as zeroth-order embedding.

In section 3 we study perturbative solutions for the embeddings. That is, we expand $\rho$, $\theta$ around the zeroth-order embedding and solve the equations (1.5). We work out the general linearized solution and find a discrete family of perturbations around the zeroth-order 
embedding. The source and vacuum expectation value for each supersymmetric combination of operators corresponding to the fluctuations can be chosen independently on one of the $\mathrm{AdS}_{3}$ making up the defect. On the remaining $\mathrm{AdS}_{3}$ the source and expectation value are then completely fixed. In particular, even with no sources turned on we find a "moduli space" of supersymmetric states. This is reminiscent of the family of supersymmetric states parametrized by the chiral condensate found for D3/D7 in [14]. At the technical level, the reason for the option to dial source and one-point function independently in both cases is that there is no constraint from the requirement for regularity at the $r=0$ slice. Physically, the different states are expected to correspond to different choices of boundary conditions for the flavor fields, at the conformal boundary of AdS. That is, the conformal boundary of $\mathrm{AdS}_{4}$ in the analysis of [14], and the boundary of $\mathrm{AdS}_{3}$ for the defect flavors studied here. We have given evidence for that interpretation in [14] which is suggestive but not conclusive. In contrast to the analysis for D3/D7, where we restricted to a more special ansatz from the outset, we find a larger family of states for D3/D5 in the current analysis. Presumably, a similarly rich family would be found for D3/D7 with a more general ansatz. For both cases, this offers interesting prospects for mutual benefits with localization calculations: to match the free energy exactly, the choice of boundary conditions corresponding to each of our embeddings has to be matched precisely. The holographic calculations can give useful intuition for what results to expect from localization calculations, and the localization results in turn could help pin down precisely which boundary conditions correspond to which embedding. We also discuss the embeddings to higher orders in the perturbative expansion, which gives some insight into the structure of the non-linear solutions.

In section 4 we analytically continue the BPS equations and embeddings for the $\mathrm{AdS}_{4}$ slicing with $\mathrm{AdS}_{3}$ defect to global Euclidean $\mathrm{AdS}_{5}$, to describe $\mathcal{N}=4 \mathrm{SYM}$ on $\mathrm{S}^{4}$, coupled to massive defect fields on an equatorial $\mathrm{S}^{3}$. The metric on the $\mathrm{AdS}_{5}$ part of the background geometry becomes

$$
g_{\mathrm{AdS}_{5}}=d R^{2}+\sinh ^{2} R\left[d \chi^{2}+\cos ^{2} \chi g_{\mathrm{S}^{3}}\right],
$$

and the embedding of the D5 branes is characterized by a bending mode $\chi$, the slipping mode $\theta$ and the worldvolume gauge field as functions of $R$ and $\beta_{1}$. The defect where the D5branes add fundamental fields is the $\mathrm{S}^{3}$ obtained as $R \rightarrow \infty$, and the massless embedding corresponds to identically vanishing $\chi, \theta$ and gauge field. We focus on a perturbative mass deformation, which corresponds to a non-trivial profile for the slipping mode. The BPS equations then require non-trivial profiles for the bending mode and gauge field as well, and they turn out to be purely imaginary for a real mass deformation. In field theory terms this reflects the fact that supersymmetric theories on $\mathrm{S}^{4}$, or by analytic continuation on $\mathrm{dS}_{4}$, in general do not satisfy respectively reflection positivity and unitarity. More specifically, the coefficient of the supersymmetry-restoring extra term that has to accompany the mass deformation on $\mathrm{AdS}_{4}$ turns imaginary upon analytic continuation to $\mathrm{dS}_{4}$ or $\mathrm{S}^{4}$. We found a similar phenomenon for the D3/D7 case studied in [14], where the gauge field turned imaginary upon analytic continuation to $\mathrm{dS}_{4}$. Unlike for the $\mathrm{AdS}_{4}$ slicing, the regularity conditions at the origin of $\mathrm{AdS}_{5}$ in the $\mathrm{S}^{4}$ slicing do fix the subleading terms of the D5-brane fields in terms of the leading terms, and the vacuum state is unique. We compute the one- 
point functions of the operators sourced by the supersymmetric combination of slipping mode, bending mode and worldvolume gauge field, and also the contribution of the defect fields to the partition function on $\mathrm{S}^{4}$ to quadratic order in the mass deformation. The result is, with the identification of holographic and field theory parameters as summarized e.g. in [29],

$$
\delta \mathcal{F}\left(\mathrm{S}^{4}\right)=-\mu \mathrm{N}_{\mathrm{f}} \mathrm{N}_{\mathrm{c}}\left[\frac{4}{3}+\frac{2 \mathrm{M}^{2}}{\mu^{2}}+\ldots\right],
$$

where $\mu=\sqrt{\lambda} / 2 \pi$ with $\lambda$ the 't Hooft coupling and $M$ is the mass of the defect fields. The dots denote subleading terms in the strong-coupling expansion and terms with higher powers of $M$. Although the partition function of $\mathcal{N}=4 \mathrm{SYM}$ on $\mathrm{S}^{4}$ is scheme dependent, we will argue that the terms at $\mathcal{O}\left(\mathrm{m}^{0}\right)$ and $\mathcal{O}\left(\mathrm{m}^{2}\right)$ in the defect contribution to the partition function are renormalization scheme independent and therefore physically meaningful quantities, that can be compared reasonably to a localization computation.

In section 5 we switch to the QFT side and compute the contribution of the defect fields to the partition function using supersymmetric localization. We are not aware of a detailed discussion of localization in theories with defects, but the matrix model can be constructed from a combination of the results obtained for four-dimensional $\mathcal{N}=4$ SYM and independent localization computations in intrinsically three-dimensional ChernSimons-matter theories. We discuss the derivation of the matrix model, for which we find

$$
\mathcal{Z}_{\text {defect }}=\int d a^{N_{c}-1} \prod_{i<j} a_{[i j]}^{2} \frac{1}{\prod_{i} \cosh ^{N_{f}}\left(\pi\left(a_{i}+M\right)\right)} e^{S_{0}}, \quad S_{0}=-\frac{8 \pi^{2}}{\lambda} N_{c} \sum_{i} a_{i}^{2} .
$$

From this matrix model we again compute the contribution of the defect fields to the partition function at strong coupling and in the quenched approximation. The result precisely matches the holographic computation resulting in (1.7). This lends strong support to the holographic computations using D3/D5, here and generally, and also to the construction of the matrix model resulting in (1.8).

\section{Reduced BPS equations for $\mathrm{AdS}_{3}$ defects}

In this section we will derive the BPS equations for general D5-brane embeddings into $\mathrm{AdS}_{5} \times \mathrm{S}^{5}$ with two copies of $\mathrm{AdS}_{3}$-sliced $\mathrm{AdS}_{4}$ as boundary geometry, which are compatible with the supersymmetries preserved by a mass deformation. We are thus looking for configurations that preserve one quarter of the original 32 supersymmetries of $\operatorname{AdS}_{5} \times \mathrm{S}^{5}$ : adding the D5-brane describing the defect fields breaks the 32 supersymmetries of the $\mathrm{AdS}_{5} \times \mathrm{S}^{5}$ solution down to 16 , and adding the mass deformation further breaks those down to 8 remaining supersymmetries. What precisely the supercharges preserved by a given embedding are is dictated by $\kappa$-symmetry [26-28]. Finding the BPS equations can be split up into two steps. First, after setting up the embedding ansatz in section 2.1, we will study embeddings with an infinitesimally small mass parameter to determine precisely which of the supersymmetries are preserved in section 2.3. With that information in hand, we will then study the full non-linear $\kappa$-symmetry constraints and derive the conditions for general embeddings to preserve the previously identified supersymmetries in section 2.4. 
We find a total of eight equations for the three functions $\rho, \theta, A_{\beta_{2}}$, which are all firstorder PDEs but not independent. We will isolate an equation which can be solved for the gauge field in section 2.6 and show that the remaining equations can be reduced to two independent ones in section 2.7 .

\subsection{Background geometry and embedding ansatz}

We want to study $\mathcal{N}=4 \mathrm{SYM}$ on two copies of $\mathrm{AdS}_{4}$, with a codimension-1 defect localized to an $\mathrm{AdS}_{3}$ slice. To realize this geometry on the boundary of $\mathrm{AdS}_{5}$, we choose coordinates where $\mathrm{AdS}_{5}$ is covered by $\mathrm{AdS}_{4}$ slices, which are in turn covered by $\mathrm{AdS}_{3}$ slices. For the $\mathrm{S}^{5}$ part it is convenient to make the $\mathrm{S}^{2}$ which the D5 branes wrap explicit. We therefore fix coordinates for the $\mathrm{AdS}_{5} \times \mathrm{S}^{5}$ background s.t.

$$
g_{\mathrm{AdS}_{5} \times \mathrm{S}^{5}}=d \rho^{2}+\cosh ^{2} \rho g_{\mathrm{AdS}_{4}}+d \theta^{2}+\cos ^{2} \theta g_{\mathrm{S}^{2}}+\sin ^{2} \theta g_{\tilde{\mathrm{S}}^{2}} .
$$

We introduce explicit coordinates for $\mathrm{AdS}_{4}$ and the two 2-spheres s.t.

$$
\begin{array}{ll}
g_{\mathrm{AdS}_{4}}=d r^{2}+\cosh ^{2} r g_{\mathrm{AdS}_{3}}, & g_{\mathrm{S}^{2}}=d \beta_{1}^{2}+\sin ^{2} \beta_{1} d \beta_{2}^{2}, \\
g_{\mathrm{AdS}_{3}}=d x^{2}+e^{2 x}\left(-d t^{2}+d y^{2}\right), & g_{\tilde{\mathrm{S}}^{2}}=d \alpha_{1}^{2}+\sin ^{2} \alpha_{1} d \alpha_{2}^{2} .
\end{array}
$$

In the probe limit the D5 branes are simply described by a DBI action with a Wess-Zumino term. With $2 \pi \alpha^{\prime}=1$ it takes the form ${ }^{2}$

$$
S_{\mathrm{D} 5}=-T_{5} \int_{\Sigma_{6}} d^{6} \xi \sqrt{-\operatorname{det}(g+F)}+T_{5} \int_{\Sigma_{6}} C_{4} \wedge F
$$

where $g$ is the pullback of the background metric. For the 4 -form gauge field we take ${ }^{3}$

$$
C_{4}=L^{-1} \zeta(\rho) \operatorname{vol}\left(\operatorname{AdS}_{4}\right)+\ldots, \quad \zeta^{\prime}(\rho)=4 \cosh ^{4} \rho .
$$

We want embeddings preserving the full $\mathrm{AdS}_{3}$ isometries and fix static gauge, using $(r, x, t, y, \vec{\beta})$ as coordinates on the D5 brane. The embedding is then described by the slipping and bending modes

$$
\theta=\theta(r, \vec{\beta}), \quad \rho=\rho(r, \vec{\beta}),
$$

respectively. The general form of the gauge field compatible with preserving the $\mathrm{AdS}_{3}$ isometries (after imposing radial gauge with $A_{r}=0$ ) is

$$
A=A_{\beta_{1}}(r, \vec{\beta}) d \beta_{1}+A_{\beta_{2}}(r, \vec{\beta}) d \beta_{2} .
$$

We expect supersymmetric embeddings to preserve a U(1), which can be used to eliminate dependence on $\beta_{2}$, as described above. For now, however, we will keep the dependence general.

\footnotetext{
${ }^{2}$ The sign convention for the WZ term is that of e.g. [26, 27, 30] and [14], and differs from the choice in $[17]$.

${ }^{3}$ This is the normalization for gauge field and the WZ term of $[17,30]$, where $d C_{4}=4 L^{-1} \operatorname{vol}\left(\operatorname{AdS}_{5}\right)$. It is different from the normalization used in [14].
} 


\subsubsection{Background Killing spinors}

For the background Killing spinors we use the $\mathrm{AdS}_{5} \times \mathrm{S}^{5}$ Killing spinor equation in the conventions of [31], namely

$$
D_{\mu} \epsilon=\frac{i}{2} \Gamma_{\mathrm{AdS}} \Gamma_{\mu} \epsilon, \quad \mu=0 \ldots 4, D_{\mu} \epsilon=\frac{i}{2} \Gamma_{\mathrm{S}^{5}} \Gamma_{\mu} \epsilon, \quad \mu=5 \ldots 9 .
$$

Whenever explicit values for indices appear, we will use an underline to distinguish local Lorentz indices from coordinate indices. We then have $\Gamma_{\text {AdS }}=\Gamma_{\underline{\rho r x t y}}=-\Gamma_{\underline{\rho r x t y}}$ and $\Gamma_{\mathrm{S}^{5}}=\Gamma \underline{\theta \alpha_{1} \alpha_{2} \beta_{1} \beta_{2}}$. The Killing spinors are given by

$$
\epsilon=R_{\mathrm{AdS}} R_{\mathrm{S}^{5}} \epsilon_{0}
$$

where the $\mathrm{AdS}_{5}$ part of the $R$-matrix, with $P_{x \pm}=\frac{1}{2}\left(\mathbb{1} \pm i \Gamma_{\underline{x}} \Gamma_{\text {AdS }}\right)$, is

$$
R_{\mathrm{AdS}}=e^{\frac{i \rho}{2} \Gamma_{\underline{\rho}} \Gamma_{\mathrm{AdS}}} e^{\frac{i r}{2} \Gamma_{\underline{r}} \Gamma_{\mathrm{AdS}}} R_{\mathrm{AdS}_{3}}, \quad R_{\mathrm{AdS}_{3}}=e^{\frac{i x}{2} \Gamma_{\underline{x}} \Gamma_{\mathrm{AdS}}}+i e^{\frac{x}{2}}\left(t \Gamma_{\underline{t}}+y \Gamma_{\underline{y}}\right) \Gamma_{\mathrm{AdS}} P_{x-} .
$$

The $S^{5}$ part, with $\Gamma_{\underline{\vec{\alpha}}}=\Gamma_{\underline{\alpha_{1}}} \Gamma_{\alpha_{2}}$ and analogously for $\Gamma_{\underline{\vec{\beta}}}$, reads

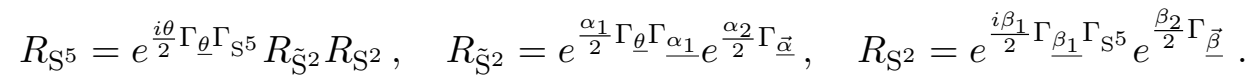

This completes the discussion of the Killing spinors. For later convenience we define chargeconjugated $R$-matrices with a tilde as $\tilde{R}_{\mathrm{AdS}}=C\left(R_{\mathrm{AdS}}\right)^{\star} C$ and analogously for the other $R$-matrices. Some useful identities are then

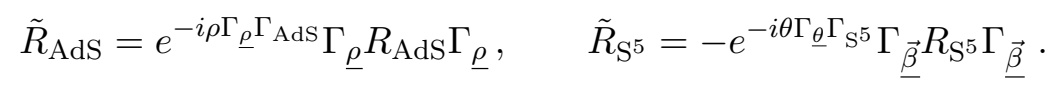

\section{$2.2 \kappa$-symmetry generalities}

The $\kappa$-symmetry condition as spelled out e.g. in [26] is a projection condition on the background Killing spinors of the form $\Gamma_{\kappa} \epsilon=\epsilon$. For an embedding to preserve some supersymmetry, the condition needs to have non-trivial solutions, and this provides the constraints which are the BPS equations. For the ansatz spelled out above

$$
\Gamma_{\kappa}=\frac{1}{\sqrt{\operatorname{det}(1+X)}}\left(J_{(5)}^{(0)}+\frac{1}{2} \gamma^{j k} F_{j k} J_{(5)}^{(1)}\right), \quad J_{(5)}^{(n)}=(-1)^{n}\left(\sigma_{3}\right)^{n+1} i \sigma_{2} \otimes \Gamma_{(0)},
$$

where $X^{i}{ }_{j}=g^{i k} F_{k j}$. Since the field strength $F$ only has non-trivial components in the $r, \vec{\beta}$ directions, the usual sum in $\Gamma_{\kappa}$ terminates after the linear term. As in [14], we switch to complex notation, such that

$$
J_{(5)}^{(0)}\left(\begin{array}{l}
\epsilon_{1} \\
\epsilon_{2}
\end{array}\right)=i C\left(\Gamma_{(0)} \epsilon\right)^{\star}, \quad J_{(5)}^{(1)}\left(\begin{array}{l}
\epsilon_{1} \\
\epsilon_{2}
\end{array}\right)=i \Gamma_{(0)} \epsilon .
$$

The $\kappa$-symmetry condition then becomes

$$
i C\left(\Gamma_{(0)} \epsilon\right)^{\star}+\frac{i}{2} \gamma^{i j} F_{i j} \Gamma_{(0)} \epsilon=\sqrt{\operatorname{det}(1+X)} \epsilon, \quad \Gamma_{(0)}=\frac{1}{6 ! \sqrt{-\operatorname{det} g}} \varepsilon^{i_{1} \ldots i_{6}} \gamma_{i_{1} \ldots i_{6}},
$$


where $\gamma_{i}=e_{i}^{a} \Gamma_{a}$ and $e^{a}=E_{\mu}^{a}\left(\partial_{i} X^{\mu}\right) d x^{i}$ is the pullback of the ten-dimensional vielbein $E^{a}$ to the D5 worldvolume. To evaluate $\Gamma_{(0)}$ we need $e^{a}$. The straightforward part is

$$
e^{\underline{\alpha_{1}}}=e^{\underline{\alpha_{2}}}=0, \quad e^{a}=E^{a}, \quad a=\underline{\beta_{1}}, \underline{\beta_{2}}, \underline{t}, \underline{y}, \underline{x}, \underline{r} .
$$

For notational convenience we introduce fraktur indices $\mathfrak{m}, \mathfrak{n}, \ldots$ running over $r, \beta_{1}, \beta_{2}$, and the remaining part then reads

$$
e^{\underline{\theta}}=d \theta=\left(\partial_{\mathfrak{m}} \theta\right) d \xi^{\mathfrak{m}}, \quad e^{\underline{\rho}}=d \rho=\left(\partial_{\mathfrak{m}} \rho\right) d \xi^{\mathfrak{m}} .
$$

With the explicit form of the pullback of the vielbein, $e^{a}$, we then find

$$
\Gamma_{(0)}=\frac{1}{\sqrt{-\operatorname{det} g}} \gamma_{r x t y \beta_{1} \beta_{2}}=\frac{\cosh ^{3} \rho \cosh ^{3} r \sqrt{-\operatorname{det} g_{\mathrm{AdS}_{3}}}}{\sqrt{-\operatorname{det} g}},
$$

where $g_{\mathrm{AdS}_{3}}$ is once again the metric on $\mathrm{AdS}_{3}$ of unit curvature radius, and $\hat{\Gamma}$ is given by

$$
\hat{\Gamma}=\Gamma_{\mathrm{AdS} 3} \gamma_{r \beta_{1} \beta_{2}}, \quad \Gamma_{\mathrm{AdS} 3}=\Gamma^{x t y}=-\Gamma_{\underline{x t y}} .
$$

The explicit expressions for the involved $\gamma$-matrices are

$$
\gamma_{\mathfrak{m}}=\Gamma_{\mathfrak{m}}+\left(\partial_{\mathfrak{m}} \rho\right) \Gamma_{\rho}+\left(\partial_{\mathfrak{m}} \theta\right) \Gamma_{\theta}
$$

Note that the $\Gamma$-matrices involve the (diagonal) $\mathrm{AdS}_{5} \times \mathrm{S}^{5}$ vielbein. The complete $\kappa$ symmetry condition then becomes

$$
i C(\hat{\Gamma} \epsilon)^{\star}+\frac{i}{2} \gamma^{i j} F_{i j} \hat{\Gamma} \epsilon=h \epsilon, \quad h=\frac{\sqrt{-\operatorname{det}(g+F)}}{\cosh ^{3} \rho \cosh ^{3} r \sqrt{-\operatorname{det} g_{\mathrm{AdS}_{3}}}} .
$$

This condition, together with the Killing spinors given previously, provides the constraints for supersymmetric embeddings and will have to be evaluated more explicitly. There are no explicit factors of $i$ in $\hat{\Gamma}$, so we can use $C^{2}=\mathbb{1}$ and $C\left(\Gamma^{\mu}\right)^{\star} C=\Gamma^{\mu}$ to rewrite it. Evaluating also $h$ more explicitly yields the for now final form of the condition

$$
i \hat{\Gamma} C \epsilon^{\star}+\frac{i}{2} \gamma^{i j} F_{i j} \hat{\Gamma} \epsilon=h \epsilon, \quad h=\sqrt{\operatorname{det}\left(g_{\mathfrak{m} \mathfrak{n}}+F_{\mathfrak{m} \mathfrak{n}}\right)},
$$

where

$$
g_{\mathfrak{m} \mathfrak{n}}=\left(g_{\mathrm{AdS}_{5} \times \mathrm{S}^{5}}\right)_{\mathfrak{m} \mathfrak{n}}+\left(\partial_{\mathfrak{m}} \rho\right)\left(\partial_{\mathfrak{n}} \rho\right)+\left(\partial_{m} \theta\right)\left(\partial_{\mathfrak{n}} \theta\right) .
$$

\subsection{Preserved supersymmetries}

In this section we determine the precise supersymmetries that are preserved by embeddings describing massive defect fields. Since the form of the supersymmetries is independent of the value of the mass parameter, we can conveniently work with an infinitesimally small mass.

We first need to determine the symmetries preserved by the massless embedding with $\rho=A=\theta=0$. Denoting the order in the mass deformation by a superscript in brackets, we have

$$
\hat{\Gamma}^{(0)}=-h^{(0)} \Gamma_{\underline{\rho}} \Gamma_{\mathrm{AdS}} \Gamma_{\underline{\vec{\beta}}}, \quad h^{(0)}=\sin \beta_{1} .
$$


Note that $\Gamma_{\text {AdS }}=\Gamma \underline{01234}=-\Gamma_{\underline{01234}}$. For the massless embedding the $\kappa$-symmetry condition (2.20) becomes

$$
i \hat{\Gamma}^{(0)} C \epsilon^{(0)^{\star}}=h^{(0)} \epsilon^{(0)} \quad \Longleftrightarrow \quad-i \Gamma_{\underline{\rho}} \Gamma_{\mathrm{AdS}} \Gamma_{\underline{\underline{\beta}}} C \epsilon^{(0)^{\star}}=\epsilon^{(0)} .
$$

With $\tilde{R}_{\mathrm{AdS}}$ and $\tilde{R}_{\mathrm{S}^{5}}$ defined below $(2.9)$, the resulting projection condition on the constant spinor $\epsilon_{0}$ in (2.7) becomes

$$
-i R_{\mathrm{AdS}}^{-1} R_{\mathrm{S}^{5}}^{-1} \Gamma_{\underline{\rho}} \Gamma_{\mathrm{AdS}} \Gamma_{\underline{\underline{\beta}}} \tilde{R}_{\mathrm{AdS}} \tilde{R}_{\mathrm{S}^{5}} C \epsilon_{0}^{\star}=\epsilon_{0} .
$$

With (2.10) and $\rho=\theta=0$, this straightforwardly evaluates to

$$
-i \Gamma_{\underline{\rho}} \Gamma_{\operatorname{AdS}} \Gamma_{\vec{\beta}} C \epsilon_{0}^{\star}=\epsilon_{0} .
$$

This condition reduces the number of preserved supersymmetries from 32 to 16 and singles out precisely which supersymmetries are preserved by the massless embedding.

To find infinitesimally massive embeddings, we solve the $\kappa$-symmetry condition $(2.20)$ at linear order in a small-fluctuation expansion around the massless embedding. We set $\theta=\theta(r)$, i.e. assume that there is no dependence on the $\mathrm{S}^{2}$ coordinates, and assume $\theta$ to be small. Similar expansions are used for $\rho$ and $A$, except for that we do not constrain their dependence on $\mathrm{S}^{2}$. Denoting by a superscript the order in the small-fluctuation expansion, the $\kappa$-symmetry condition at linear order then reads

$$
i \hat{\Gamma}^{(1)} C \epsilon^{(0)^{\star}}+i \hat{\Gamma}^{(0)} C \epsilon^{(1)^{\star}}+\frac{i}{2} \gamma^{(0) i j} F_{i j}^{(1)} \hat{\Gamma}^{(0)} \epsilon^{(0)}=h^{(0)} \epsilon^{(1)} .
$$

On the right hand side we have used that corrections to $h$ are at least quadratic, so $h^{(1)}=0$. The Killing spinor at linearized order straightforwardly evaluates to

$$
\epsilon^{(1)}=\frac{i}{2}\left(\rho \Gamma_{\underline{\rho}} \Gamma_{\mathrm{AdS}}+\theta \Gamma_{\underline{\theta}} \Gamma_{\mathrm{S}^{5}}\right) \epsilon^{(0)}=: \delta R \epsilon^{(0)} .
$$

Noting that $C(\delta R)^{\star} C=-\delta R$, the $\kappa$-symmetry condition (2.26) becomes

$$
i\left(\hat{\Gamma}^{(1)}-\hat{\Gamma}^{(0)} \delta R\right) C \epsilon^{(0)^{\star}}+\frac{i}{2} \gamma^{(0) i j} F_{i j}^{(1)} \hat{\Gamma}^{(0)} \epsilon^{(0)}=h^{(0)} \delta R \epsilon^{(0)} .
$$

Using $\left(\hat{\Gamma}^{(0)}\right)^{2}=h^{(0)^{2}} \mathbb{1}$, the massless projection condition can be written as $C \epsilon^{(0)^{\star}}=$ $-i \hat{\Gamma}^{(0)} \epsilon^{(0)} / h^{(0)}$. So we can eliminate $C \epsilon^{(0)^{\star}}$ and the $\kappa$-symmetry condition becomes

$$
\frac{1}{h^{(0)}}\left(\hat{\Gamma}^{(1)}-\hat{\Gamma}^{(0)} \delta R\right) \hat{\Gamma}^{(0)} \epsilon^{(0)}+\frac{i}{2} \gamma^{(0) i j} F_{i j}^{(1)} \hat{\Gamma}^{(0)} \epsilon^{(0)}=h^{(0)} \delta R \epsilon^{(0)}
$$

We can now use $\hat{\Gamma}^{(0)} \delta R \hat{\Gamma}^{(0)}=h^{(0)^{2}} \delta R$ to find

$$
\frac{1}{h^{(0)}} \hat{\Gamma}^{(1)} \hat{\Gamma}^{(0)} \epsilon^{(0)}+\frac{i}{2} \gamma^{(0) i j} F_{i j}^{(1)} \hat{\Gamma}^{(0)} \epsilon^{(0)}=2 h^{(0)} \delta R \epsilon^{(0)} .
$$

For the computation of the term involving the field strength it is useful to note that $\gamma^{(0) i j} F_{i j}=\Gamma^{i j} F_{i j}$, since for the zeroth-order embedding with $\rho=\theta=0$ the induced metric 
$g$ coincides with the corresponding part of $g_{\mathrm{AdS}_{5} \times \mathrm{S}^{5}}$ for the components of interest. We will also set

$$
A_{\beta_{i}}=f(r) \omega_{i}(\vec{\beta})
$$

such that $A=f \omega$ with $\omega$ a one-form on $\mathrm{S}^{2}$. For this subsection we use the shorthand notation $\partial_{\beta_{1 / 2}}=\partial_{1 / 2}$ and a prime to denote derivatives w.r.t. $r$. Using once again that $\rho=\theta=0$ for the zeroth-order embedding, we then find

$$
\frac{1}{2} \gamma^{(0) i j} F_{i j}^{(1)}=f^{\prime} \Gamma_{\underline{r}}\left(\omega_{1} \Gamma_{\underline{\beta_{1}}}+\omega_{2} \csc \beta_{1} \Gamma_{\underline{\beta_{2}}}\right)+f(\star d \omega) \Gamma_{\underline{\vec{\beta}}},
$$

where $\star d \omega=\csc \beta_{1}\left(\partial_{1} \omega_{2}-\partial_{2} \omega_{1}\right)$. For the combination which appears in (2.30) this yields

$$
\frac{i}{2} \gamma^{(0) i j} F_{i j}^{(1)} \hat{\Gamma}^{(0)}=i\left[f^{\prime} \Gamma_{\underline{r}}\left(\omega_{2} \Gamma_{\underline{\beta_{1}}}-\sin \beta_{1} \omega_{1} \Gamma_{\underline{\beta_{2}}}\right)+f \sin \beta_{1}(\star d \omega) \mathbb{1}\right] \Gamma_{\underline{\rho}} \Gamma_{\text {AdS }} .
$$

The last object we have to work out explicitly is $\hat{\Gamma}^{(1)}$ with $\hat{\Gamma}$ given in (2.17). The combination appearing in (2.30) becomes

$$
\frac{1}{h^{(0)}} \hat{\Gamma}^{(1)} \hat{\Gamma}^{(0)}=\sin \beta_{1}\left(\theta^{\prime} \Gamma_{\underline{\theta}}+\rho^{\prime} \Gamma_{\underline{\rho}}\right) \Gamma_{\underline{\underline{r}}}+\sin \beta_{1}\left(\partial_{1} \rho\right) \Gamma_{\underline{\rho}} \Gamma_{\underline{\beta_{1}}}+\left(\partial_{2} \rho\right) \Gamma_{\underline{\rho}} \Gamma_{\underline{\beta_{2}}} .
$$

The $\kappa$-symmetry condition in eq. (2.30), after dividing by $\sin \beta_{1}$, then takes the explicit form

$$
\begin{aligned}
0= & {\left[\theta^{\prime} \Gamma_{\underline{\theta}} \Gamma_{\underline{r}}+\rho^{\prime} \Gamma_{\underline{\rho}} \Gamma_{\underline{r}}+\left(\partial_{1} \rho\right) \Gamma_{\underline{\rho}} \Gamma_{\beta_{1}}+\csc \beta_{1}\left(\partial_{2} \rho\right) \Gamma_{\underline{\rho}} \Gamma_{\beta_{2}}-i \rho \Gamma_{\underline{\rho}} \Gamma_{\text {AdS }}-i \theta \Gamma_{\underline{\theta}} \Gamma_{\mathrm{S}^{5}}\right.} \\
& \left.-i f^{\prime}\left(\omega_{1} \Gamma_{\underline{\beta_{2}}}-\omega_{2} \csc \beta_{1} \Gamma_{\underline{\beta_{1}}}\right) \Gamma_{\underline{\rho}} \Gamma_{\underline{r}} \Gamma_{\text {AdS }}+i f(\star d \omega) \Gamma_{\underline{\rho}} \Gamma_{\text {AdS }}\right] \epsilon^{(0)} .
\end{aligned}
$$

Multiplying by $\Gamma_{\underline{\theta}} \Gamma_{\underline{\rho}}$ and using that $\epsilon_{0}$ is a chiral spinor with $\Gamma_{11} \epsilon_{0}=\epsilon_{0} \Leftrightarrow \Gamma_{\mathrm{S}^{5}} \epsilon=-\Gamma_{\mathrm{AdS}} \epsilon$, yields

$$
\begin{aligned}
{\left[\theta^{\prime} \Gamma_{\underline{\rho}} \Gamma_{\underline{r}}+i \theta \Gamma_{\underline{\rho}} \Gamma_{\text {AdS }}\right] \epsilon^{(0)}=} & {\left[i(\rho-f \star d \omega) \Gamma_{\text {AdS }}-\left(\partial_{1} \rho\right) \Gamma_{\underline{\beta_{1}}}-\csc \beta_{1}\left(\partial_{2} \rho\right) \Gamma_{\underline{\beta_{2}}}\right] \Gamma_{\underline{\theta}} \epsilon^{(0)} } \\
& +\left[i f^{\prime}\left(\omega_{2} \csc \beta_{1} \Gamma_{\underline{\beta_{1}}}-\omega_{1} \Gamma_{\underline{\beta_{2}}}\right) \Gamma_{\underline{r}} \Gamma_{\text {AdS }}-\rho^{\prime} \Gamma_{\underline{r}}\right] \Gamma_{\underline{\theta}} \epsilon^{(0)}
\end{aligned}
$$

The term on the left hand side has no non-trivial dependence on the $S^{5}$ coordinates. That is, after multiplying both sides of the equation by $R_{\mathrm{S}^{5}}^{-1}$ all dependence drops out. The first term on the right hand side only has dependence on the $\mathrm{AdS}_{5}$ directions through $\rho$ and $f$, no non-trivial $\mathrm{AdS}_{5} \Gamma$-matrix structures. That means it is independent of the $\mathrm{AdS}_{3}$ directions $x, t, y$ after multiplying both sides of the equation by $R_{\mathrm{AdS}}^{-1}$. The interesting term is the second one on the right hand side, in line two. It has non-trivial dependence on the $\mathrm{S}^{5}$ and on the $\mathrm{AdS}_{3}$ directions, due to the appearance of $\Gamma_{\underline{r}} .{ }^{4}$ That means it either has to vanish by itself, or at least one of the non-trivial dependences on the $\mathrm{S}^{5}$ and the $\mathrm{AdS}_{3}$ directions has to drop out for it to cancel with one of the other terms in the equation. But it is not possible to cancel the non-trivial $\mathrm{AdS}_{3}$ dependence, so it must be the dependence on the $\mathrm{S}^{5}$ directions which cancels. This allows it to combine with the term on the left hand side, which also has non-trivial dependence on the $\mathrm{AdS}_{3}$ directions. In fact, all of the $\Gamma$-matrix structures in $R_{\mathrm{AdS}}^{-1} \Gamma_{\underline{r}} R_{\mathrm{AdS}}$ have non-trivial dependence on the $\mathrm{AdS}_{3}$ directions. So in the first term on the right hand side, which does not have such dependence, the $S^{5}$ dependence has to cancel as well.

\footnotetext{
${ }^{4}$ At $\rho=0, R_{\mathrm{AdS}}^{-1} \Gamma_{\underline{r}} R_{\mathrm{AdS}}$ has four independent $\Gamma$-matrix structures whose coefficients depend on $(x, t, y)$ in such a way that the dependence can not be cancelled by imposing a projector on $\epsilon_{0}$. See (2.50a) below.
} 


\subsubsection{Solving for $\mathrm{S}^{2}$ dependences}

From the fact that the $\mathrm{S}^{5}$ dependence in the first term on the right hand side of (2.36) has to cancel, we now see that either $f$ and $\rho$ have to have the same dependence on $r$ up to overall constants, or $\star d \omega$ has to be constant. The first option is the one leading to non-trivial results and we thus write $\rho=f \psi$, where $f$ is the radial profile that appeared already in (2.31). Note that the relative normalization of $\omega$ and $\psi$ now matters - they can not both be chosen normalized w.o.l.g. at the same time. Using also $\Gamma_{11} \epsilon^{(0)}=\epsilon^{(0)}$ to convert $\Gamma_{\text {AdS }}$ to $\Gamma_{\mathrm{S}^{5}}$, we then find

$$
\begin{aligned}
{\left[\theta^{\prime} \Gamma_{\underline{\rho}} \Gamma_{\underline{r}}+i \theta \Gamma_{\underline{\rho}} \Gamma_{\text {AdS }}\right] \epsilon^{(0)}=} & f\left[i(\psi-\star d \omega) \Gamma_{S^{5}}-\left(\partial_{1} \psi\right) \Gamma_{\underline{\beta_{1}}}-\csc \beta_{1}\left(\partial_{2} \psi\right) \Gamma_{\underline{\beta_{2}}}\right] \Gamma_{\underline{\theta}} \epsilon^{(0)} \\
& +f^{\prime}\left[i \omega_{2} \csc \beta_{1} \Gamma_{\underline{\beta_{1}}}-i \omega_{1} \Gamma_{\underline{\beta_{2}}}+\psi \Gamma_{\mathrm{S}^{5}}\right] \Gamma_{\underline{\theta}} \Gamma_{\underline{r}} \Gamma_{\mathrm{AdS}} \epsilon^{(0)}
\end{aligned}
$$

We start with the second term on the right hand side, in which all $S^{5}$ dependence has to drop out after multiplying by $R_{\mathrm{S}^{5}}^{-1}$, as argued above. Note that this term is algebraic in $\omega$ and $\psi$. To make the structure more apparent, we rewrite (2.37) as

$$
\left[\theta^{\prime} \Gamma_{\underline{\rho}} \Gamma_{\underline{r}}+i \theta \Gamma_{\underline{\rho}} \Gamma_{\mathrm{AdS}}\right] \epsilon^{(0)}=f Y \epsilon^{(0)}+f^{\prime} Z \Gamma_{\underline{r}} \Gamma_{\mathrm{AdS}} \epsilon^{(0)}
$$

where $Y$ and $Z$ are independent of $r$, and are given by

$$
\begin{aligned}
Y & =\left[i(\psi-\star d \omega) \Gamma_{\mathrm{S}^{5}}-\left(\partial_{1} \psi\right) \Gamma_{\underline{\beta_{1}}}-\csc \beta_{1}\left(\partial_{2} \psi\right) \Gamma_{\underline{\beta_{2}}}\right] \Gamma_{\underline{\theta}}, \\
Z & =\left[i \omega_{2} \csc \beta_{1} \Gamma_{\underline{\beta_{1}}}-i \omega_{1} \Gamma_{\underline{\beta_{2}}}+\psi \Gamma_{\mathrm{S}^{5}}\right] \Gamma_{\underline{\theta}} .
\end{aligned}
$$

So the task is to find $\psi, \omega$ such that $R_{\mathrm{S}^{5}}^{-1} Z R_{\mathrm{S}^{5}}$ is independent of the $\mathrm{S}^{5}$ coordinates. We can see immediately that $R_{\mathrm{S}^{5}}^{-1} Y R_{\mathrm{S}^{5}}$ and $R_{\mathrm{S}^{5}}^{-1} Z R_{\mathrm{S}^{5}}$ will involve the same Clifford algebra structures. For the explicit evaluation we will fix the position at which the D5-branes are located on the $\tilde{S}^{2}$ in (2.1) to $\alpha_{1}=0$. This can be done without loss of generality, since we can arbitrarily choose which point corresponds to the north pole in the coordinates $\alpha_{1}, \alpha_{2}$. At $\theta=0$ we then find

$$
\begin{aligned}
& R_{\mathrm{S}^{5}}^{-1} \Gamma_{\underline{\beta_{1}}} \Gamma_{\underline{\theta}} R_{\mathrm{S}^{5}}=\cos \beta_{1}\left(\cos \beta_{2} \Gamma_{\underline{\beta_{1}}}+\sin \beta_{2} \Gamma_{\underline{\beta_{2}}}\right) \Gamma_{\underline{\theta}}-\sin \beta_{1} \Gamma_{\mathrm{S}^{5}} \Gamma_{\underline{\theta}}, \\
& R_{\mathrm{S}^{5}}^{-1} \Gamma_{\beta_{2}} \Gamma_{\underline{\theta}} R_{\mathrm{S}^{5}}=\left(\cos \beta_{2} \Gamma_{\underline{\beta_{2}}}-\sin \beta_{2} \Gamma_{\underline{\beta_{1}}}\right) \Gamma_{\underline{\theta}}, \\
& R_{\mathrm{S}^{5}}^{-1} \Gamma_{\mathrm{S}^{5}} \Gamma_{\underline{\theta}} R_{\mathrm{S}^{5}}=\cos \beta_{1} \Gamma_{\mathrm{S}^{5}} \Gamma_{\underline{\theta}}-i \sin \beta_{1}\left(\cos \beta_{2} \Gamma_{\underline{\beta_{1}}}+\sin \beta_{2} \Gamma_{\underline{\beta_{2}}}\right) \Gamma_{\underline{\theta}} \text {. }
\end{aligned}
$$

The expressions involve three independent Clifford algebra structures, and to eliminate the $\mathrm{S}^{5}$ dependence we have to solve

$$
R_{\mathrm{S}^{5}}^{-1} Z R_{\mathrm{S}^{5}}=\left(i c_{1} \Gamma_{\underline{\beta_{1}}}+i c_{2} \Gamma_{\underline{\beta_{2}}}+c_{3} \Gamma_{\mathrm{S}^{5}}\right) \Gamma_{\underline{\theta}},
$$

with generically complex constants $c_{1}, c_{2}, c_{3}$. This is a system of three linear equations for $\omega_{i}$ and $\psi$, and can be solved straightforwardly. Demanding $R_{\mathrm{S}^{5}}^{-1} Y R_{\mathrm{S}^{5}}$ to be independent of 
the position on $\mathrm{S}^{5}$ as well adds another three equations, overconstraining the system. The system we get from (2.41) is

$$
\left(\begin{array}{ccc}
-\sin \beta_{1} \cos \beta_{2} & \sin \beta_{2} & \cot \beta_{1} \cos \beta_{2} \\
-\sin \beta_{1} \sin \beta_{2} & -\cos \beta_{2} & \cot \beta_{1} \sin \beta_{2} \\
\cos \beta_{1} & 0 & 1
\end{array}\right)\left(\begin{array}{l}
\psi \\
\omega_{1} \\
\omega_{2}
\end{array}\right)=\left(\begin{array}{l}
c_{1} \\
c_{2} \\
c_{3}
\end{array}\right) \text {. }
$$

For all $c_{i}$ real the solutions are real, and we shall assume that henceforth. The general solution reads

$$
\begin{aligned}
\psi & =c_{3} \cos \beta_{1}-\sin \beta_{1}\left(c_{1} \cos \beta_{2}+c_{2} \sin \beta_{2}\right), \\
\omega_{1} & =c_{1} \sin \beta_{2}-c_{2} \cos \beta_{2}, \\
\omega_{2} & =\sin \beta_{1}\left(\cos \beta_{1}\left(c_{1} \cos \beta_{2}+c_{2} \sin \beta_{2}\right)+c_{3} \sin \beta_{1}\right) .
\end{aligned}
$$

It satisfies $\triangle_{\mathrm{S}^{2}} \psi=-2 \psi$, so $\psi$ is an $\ell=1$ mode. Furthermore, $\omega_{i}=\sqrt{g_{\mathrm{S}^{2}}} \varepsilon_{i j} g_{\mathrm{S}^{2}}^{j k} \partial_{k} \psi$ with $\varepsilon_{12}=1$, so we also have $\psi=\frac{1}{2} \star d \omega$. For $c_{1}=c_{2}=0$ there is no $\beta_{2}$ dependence and translations in $\beta_{2}$ are the preserved $\mathrm{U}(1)$. Finally, we have to verify that this solution also makes $R_{\mathrm{S}^{5}}^{-1} Y R_{\mathrm{S}^{5}}$ independent of the $\mathrm{S}^{5}$ coordinates. Indeed, using (2.43) results in

$$
R_{\mathrm{S}^{5}}^{-1} Y R_{\mathrm{S}^{5}}=-i R_{\mathrm{S}^{5}}^{-1} Z R_{\mathrm{S}^{5}}
$$

So the $\mathrm{S}^{5}$ dependence drops out in the first term on the right hand side of (2.38) as well, as desired.

\subsubsection{Solving for the radial profiles}

With the results of the previous section we have eliminated all non-trivial dependence on the $\mathrm{S}^{5}$ and can focus on the $\mathrm{AdS}_{5}$ part. This will yield the explicit form of the necessary projector on the Killing spinor, singling out the preserved supersymmetries, and the linearized BPS equations for infinitesimally massive embeddings. Eq. (2.38), after multiplying both sides by $R_{\mathrm{S}^{5}}^{-1}$ and using $(2.43)$, becomes

$$
\left[\theta^{\prime} \Gamma_{\underline{\rho}} \Gamma_{\underline{r}}+i \theta \Gamma_{\underline{\rho}} \Gamma_{\mathrm{AdS}}\right] R_{\mathrm{AdS}}^{(0)} \epsilon_{0}=\left[f \mathbb{1}+i f^{\prime} \Gamma_{\underline{r}} \Gamma_{\mathrm{AdS}}\right] R_{\mathrm{AdS}}^{(0)} \Gamma_{p} \epsilon_{0},
$$

where we have defined

$$
\Gamma_{p}=\left(c_{1} \Gamma_{\underline{\beta_{1}}}+c_{2} \Gamma_{\underline{\beta_{2}}}-i c_{3} \Gamma_{\mathrm{S}^{5}}\right) \Gamma_{\underline{\theta}} .
$$

Note that $\Gamma_{p}^{2}=-|c|^{2} \mathbb{1}$ with $|c|^{2}=c_{1}^{2}+c_{2}^{2}+c_{3}^{2}$, and $\Gamma_{p}$ commutes with $\operatorname{AdS}_{5} \Gamma$-matrices. So it can be used straightforwardly for constructing projectors.

To solve (2.45) systematically we turn to the dependence on the $\mathrm{AdS}_{3}$ directions $(x, t, y)$. We introduce an operator $\mathcal{R}_{A}$ and for later convenience also $\mathcal{R}_{S}$, defined by

$$
\mathcal{R}_{A}[\Gamma]=R_{\mathrm{AdS}}^{-1} \Gamma R_{\mathrm{AdS}}, \quad \mathcal{R}_{S}[\Gamma]=R_{\mathrm{S}^{5}}^{-1} \Gamma R_{\mathrm{S}^{5}} .
$$

At $\rho=0$, we then note the useful identity

$$
\mathcal{R}_{A}^{(0)}\left[\Gamma_{\underline{\rho}} \Gamma_{\underline{r}}\right]=i \tanh r \mathcal{R}_{A}^{(0)}\left[\Gamma_{\underline{\rho}} \Gamma_{\mathrm{AdS}}\right]+\operatorname{sech} r \Gamma_{\underline{\rho}} \Gamma_{\underline{r}} .
$$


Multiplying (2.45) by $\left(R_{\text {AdS }}^{(0)}\right)^{-1}$, we then find that the $\kappa$-symmetry condition becomes

$$
i\left(\theta^{\prime} \tanh r+\theta\right) \mathcal{R}_{A}^{(0)}\left[\Gamma_{\underline{\rho}} \Gamma_{\text {AdS }}\right] \epsilon_{0}+\theta^{\prime} \operatorname{sech} r \Gamma_{\underline{\rho}} \Gamma_{\underline{r}}=f \Gamma_{p} \epsilon_{0}+i f^{\prime} \mathcal{R}_{A}^{(0)}\left[\Gamma_{\underline{r}} \Gamma_{\text {AdS }}\right] \Gamma_{p} \epsilon_{0} .
$$

The parts without $\mathcal{R}_{A}$ already suggest a form of the projector to impose, but since they may be modified by contributions from the terms involving $\mathcal{R}_{A}$, we need to analyze the $(x, t, y)$ dependent terms to derive the projector. The explicit identities we will use for that are

$$
\mathcal{R}_{A}^{(0)}\left[\Gamma_{\underline{\underline{r}}} \Gamma_{\mathrm{AdS}}\right]=i e^{x}\left(y \Gamma_{\underline{y}}+t \Gamma_{\underline{t}}\right) \Gamma_{\underline{r}}-i\left(e^{x}\left(y^{2}-t^{2}-1\right)+e^{-x}\right) \Gamma_{\underline{x}} P_{x+} \Gamma_{\underline{r}}+e^{x} \Gamma_{\underline{r}} \Gamma_{\mathrm{AdS}},
$$

$\mathcal{R}_{A}^{(0)}\left[\Gamma_{\underline{\rho}} \Gamma_{\text {AdS }}\right] \Gamma_{\underline{\rho}} \Gamma_{\underline{r}}=\cosh r \mathcal{R}_{A}^{(0)}\left[\Gamma_{\underline{r}} \Gamma_{\text {AdS }}\right]+i \sinh r \mathbb{1}$

The first one shows that the $\mathrm{AdS}_{3}$ dependence can not be canceled within $\mathcal{R}_{A}^{(0)}\left[\Gamma_{\underline{\rho}} \Gamma_{\text {AdS }}\right]$ and $R_{A}^{(0)}\left[\Gamma_{\underline{r}} \Gamma_{\text {AdS }}\right]$ separately, since there are too many independent Clifford algebra structures with non-trivial dependence. The second identity shows precisely which projector has to be imposed on $\epsilon_{0}$ to have a chance to cancel the non-trivial $\mathrm{AdS}_{3}$ dependence between the two terms. Using it in (2.49) gives

$i \mathcal{R}_{A}^{(0)}\left[\Gamma_{\underline{\rho}} \Gamma_{\text {AdS }}\right]\left(\theta^{\prime} \tanh r+\theta-f^{\prime} \operatorname{sech} r \Gamma_{\underline{\rho}} \Gamma_{\underline{r}} \Gamma_{p}\right) \epsilon_{0}=\left(f+f^{\prime} \tanh r\right) \Gamma_{p} \epsilon_{0}-\theta^{\prime} \operatorname{sech} r \Gamma_{\underline{\rho}} \Gamma_{\underline{r}} \epsilon_{0}$.

From this equation we can finally read off the projector, from the requirement to make the left hand side cancel in a non-trivial way. This yields

$$
\frac{1}{|c|} \Gamma_{\underline{\rho}} \Gamma_{\underline{r}} \Gamma_{p} \epsilon_{0}=\lambda \epsilon_{0}, \quad \lambda= \pm 1,
$$

with $\Gamma_{p}$ defined in (2.46). With this projector the equation for $\kappa$-symmetry in $(2.51)$ becomes

$i \mathcal{R}_{A}^{(0)}\left[\Gamma_{\underline{\rho}} \Gamma_{\text {AdS }}\right]\left(\theta^{\prime} \tanh r+\theta-\lambda|c| f^{\prime} \operatorname{sech} r\right) \epsilon_{0}=-\left(\theta^{\prime} \operatorname{sech} r+\lambda|c|\left(f+f^{\prime} \tanh r\right)\right) \Gamma_{\underline{\rho}} \Gamma_{\underline{r}} \epsilon_{0}$.

The terms in brackets on each side now have to vanish separately and we obtain two $1^{\text {st }}$-order equations for the slipping and bending modes

$$
\theta^{\prime} \tanh r+\theta-\lambda|c| f^{\prime} \operatorname{sech} r=0, \quad \theta^{\prime} \operatorname{sech} r+\lambda|c|\left(f+f^{\prime} \tanh r\right)=0 .
$$

They have non-trivial solutions and we have verified that these two equations indeed imply the linearized equations of motion derived from the action in (2.2).

\subsection{Non-linear $\kappa$-symmetry}

In this section we derive the form of the fully non-linear $\kappa$-symmetry equations for embeddings preserving the eight supersymmetries that are preserved by massive defect fields. We have the general form of the $\kappa$-symmetry condition in (2.20) and the two projection conditions on the background Killing spinors derived in the previous section and given 
in (2.25) and (2.52). Note that the projection conditions in (2.25) and (2.52) restrict the constant spinor $\epsilon_{0}$ that parametrizes the $\mathrm{AdS}_{5} \times \mathrm{S}^{5}$ Killing spinors via (2.7). The conditions therefore carry over directly from the infinitesimally massive case to the general case considered now, where the $R$-matrices in (2.7) evaluated at the location of the D5-branes may take a different form.

We start out from (2.20) and handle the first term in that equation first. With the definition of the charge conjugated $R$-matrices in (2.10) we find

$$
C \epsilon^{\star}=-e^{-i \rho \Gamma_{\underline{\rho}} \Gamma_{\mathrm{AdS}}} e^{-i \theta \Gamma_{\underline{\theta}} \Gamma_{\mathrm{S}} 5} \Gamma_{\underline{\rho}} \Gamma_{\underline{\vec{\beta}}} R_{\mathrm{AdS}} R_{\mathrm{S}^{5}} \Gamma_{\underline{\rho}} \Gamma_{\vec{\beta}} C\left(\epsilon_{0}\right)^{\star} .
$$

We can now use the massless projection condition (2.25) to eliminate the charge-conjugated constant spinor, and find

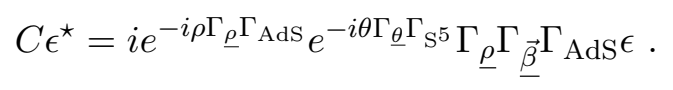

The $\kappa$-symmetry condition (2.20) then involves no more charge conjugation and becomes

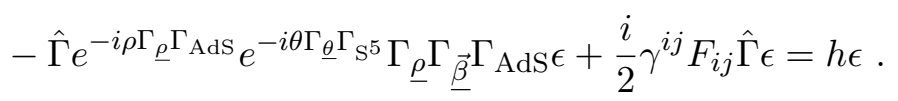

For the gauge field term it is convenient to use the field strength with indices raised by the induced metric on the D5-brane worldvolume, and write it as

$$
\frac{1}{2} \gamma^{i j} F_{i j}=\gamma_{r \beta_{i}} F^{r \beta_{i}}+\gamma_{\beta_{1} \beta_{2}} F^{\beta_{1} \beta_{2}}
$$

The remaining task is to use the massive projector in (2.52) to derive an explicit form for the conditions implied by the constraint (2.57). This is a cumbersome but straightforward task, and we give the details in appendix A. The result is that, after multiplying eq. (2.57) by $R_{\mathrm{AdS}}^{-1} R_{\mathrm{S}^{5}}^{-1}$, the condition can be written as

$$
\mathcal{Q}_{\mathcal{K}} \mathcal{K} \Gamma_{\underline{\rho}} \epsilon_{0}+\mathcal{Q}_{\mathbb{1}} \epsilon_{0}=h \epsilon_{0},
$$

where $\mathcal{K}$ is a matrix with explicit dependence on the $\mathrm{AdS}_{3}$ coordinates, and the explicit form of $\mathcal{Q}_{\mathcal{K}}$ and $\mathcal{Q}_{\mathbb{1}}$ can be found in appendix A. The crucial point is that the available projectors and chirality constraints have been implemented completely, such that $\mathcal{Q}_{\mathcal{K}}$ and $\mathcal{Q}_{1}$ necessarily have to vanish separately for the $\kappa$-symmetry condition to be satisfied. We thus have to solve

$$
\mathcal{Q}_{\mathcal{K}}=0, \quad \mathcal{Q}_{\mathbb{1}}-h \mathbb{1}=0 .
$$

We will discuss the explicit form of the implied equations in the next section.

\subsection{The BPS equations}

In this section we spell out explicitly the equations resulting from the two matrix equations in (2.60). For the massive projector in (2.52) we left the choice of $c_{1}, c_{2}$ and $c_{3}$ arbitrary up to this point. But we shall from now on set

$$
c_{1}=c_{2}=0, \quad c_{3}=1 .
$$


There is no explicit dependence on $\beta_{2}$ in the metric and with this choice of projector the Clifford algebra manipulations do not introduce dependence either, such that the U(1) isometry remaining from the $\mathrm{S}^{2}$ that the D5-branes wrap in $\mathrm{S}^{5}$ is realized as translations in $\beta_{2}$. The equations can then be solved with only trivial dependence on $\beta_{2}$. Moreover, the first equation in (2.60) in particular implies $\operatorname{tr}\left(\tilde{\mathcal{Q}}_{\mathcal{K}} \Gamma_{\theta \beta_{1} \beta_{2}}\right)=0$, and with the specific choice of projector in (2.61) this condition implies

$$
F_{r \beta_{1}}=0
$$

That means $A_{\beta_{1}}$ is independent of $r$ and depends on $\beta_{1}$ only, and can be set to zero by a residual gauge transformation.

To conveniently write the remaining equations resulting from (2.60) we change coordinates to

$$
r=2 \tanh ^{-1} \tan \frac{z}{2}, \quad \beta_{1}=\cos ^{-1} x
$$

such that $z \in\left(-\frac{\pi}{2}, \frac{\pi}{2}\right)$ to completely cover $\mathrm{AdS}_{5}$, and $x \in[0,1]$. As shorthands we also introduce

$$
F_{z}:=F_{z \beta_{2}}=\frac{\partial r}{\partial z} F_{r \beta_{2}}, \quad \quad F_{x}:=F_{x \beta_{2}}=\frac{\partial \beta_{1}}{\partial x} F_{\beta_{1} \beta_{2}} .
$$

Moreover, with $\mathfrak{a}, \mathfrak{b}=z, x$ and $\epsilon^{z x}=1$, we define

$$
B_{1}=\varepsilon^{\mathfrak{a} \mathfrak{b}}\left(\partial_{\mathfrak{a}} \theta\right) F_{\mathfrak{b}}, \quad B_{2}=\varepsilon^{\mathfrak{a} \mathfrak{b}}\left(\partial_{\mathfrak{a}} \rho\right) F_{\mathfrak{b}}, \quad B_{3}=\varepsilon^{\mathfrak{a} \mathfrak{b}}\left(\partial_{\mathfrak{a}} \rho\right) \partial_{\mathfrak{b}} \theta
$$

and

$$
\begin{array}{ll}
A_{1}=\partial_{x}(\sinh \rho \cos \theta), & A_{2}=\partial_{x}(\sinh \rho \sin \theta), \\
A_{3}=\operatorname{sech} \rho\left(\lambda x \partial_{z} \theta+\tan z \partial_{z} \rho\right)-\sec ^{2} z \sinh \rho, & A_{4}=\partial_{x}(\cosh \rho \cos \theta), \\
A_{5}=\partial_{x}(\cosh \rho \sin \theta) . &
\end{array}
$$

From $\mathcal{Q}_{\mathcal{K}}=0$ we find four equations, which, with the shorthands defined above, take the form

$$
\cos ^{2} \theta\left(\sin \theta A_{3}+\lambda \tanh \rho F_{z}\right)+\tan z B_{1}-\lambda x B_{2}=0
$$

$$
\begin{array}{r}
\lambda \partial_{z} \sin \theta+\sin \theta \tan z B_{3}-\lambda \sinh \rho B_{1}+\cosh \rho\left[\frac{F_{z} \tan z}{1-x^{2}}-\lambda \tan \theta B_{2}-\sec ^{2} z A_{1}\right]=0 \\
\lambda \sinh \rho\left[\frac{x F_{z}}{1-x^{2}}-\tan \theta B_{1}\right]-\tan z \cos \theta B_{3}+\cosh \rho\left(\lambda B_{2}+\sec ^{2} z A_{2}\right)=0 \\
\cos ^{2} \theta\left(\cos \theta A_{3}-\lambda \tan \theta \tanh \rho F_{z}\right)-\tanh \rho\left(\lambda x B_{1}+\tan z B_{2}\right)-\sec ^{2} z F_{x}=0
\end{array}
$$


We similarly find four equations resulting from $\mathcal{Q}_{\mathbb{1}}-h \mathbb{1}=0$, and those read

$$
\begin{aligned}
-\frac{h}{\sqrt{1-x^{2}}}+\cos \theta \cosh ^{2} \rho\left[\cos ^{2} \theta-\lambda \tanh \rho \tan z\left(\left(1-x^{2}\right) \partial_{x} \theta+\frac{x}{2} \sin 2 \theta\right)\right] & \\
+\frac{\lambda}{2} \sin 2 \theta\left(x \cos \theta \partial_{z} \rho+\cosh \rho F_{z}\right)+\lambda x \cosh \rho B_{1}+\lambda\left(1-x^{2}\right) \cos \theta B_{3}=0, & =0 \\
\cosh \rho\left[\frac{x F_{z}}{1-x^{2}}-\tan \theta B_{1}+\tan z\left(F_{x}+x A_{1}+\sinh \rho \cos \theta\right)+\lambda A_{5}\right] & -\cos \theta \partial_{z} \rho+\sinh \rho B_{2}+x \sin \theta B_{3}=0, \\
\lambda \tan z\left(\tan \theta F_{x}+x A_{2}\right)-A_{4}+\lambda\left(\tan \theta \tanh \rho B_{2}+B_{1}-x \operatorname{sech} \rho \cos \theta B_{3}\right) & =0, \\
\lambda x \cos ^{3} \theta\left(\operatorname{sech} \rho \partial_{z} \rho-\tan z \sinh \rho\right)-\lambda x \tanh \rho B_{2} & =0 . \\
+\cos ^{2} \theta\left(\lambda F_{z}-\sin \theta \cosh \rho\right)+\lambda \tan z\left(\left(1-x^{2}\right) \cos \theta \partial_{x} \sinh \rho-x F_{x}\right) & =0 .
\end{aligned}
$$

We therefore have eight equations for two functions $\rho, \theta$ and the two field strength components $F_{r \beta_{2}}, F_{\beta_{1} \beta_{2}}$, which in addition have to satisfy the Bianchi identity for $F$. The equations are clearly non-linear (in the functions and in derivative terms), and we have a square root implicit in the definition of $h$ in eq. (2.68a). However, except for (2.68a) the equations are only quadratic in derivative terms, and since we have more equations than functions we may attempt to derive quasilinear equations by taking combinations of these equations. This will be done in the next section.

\subsection{Solving for the gauge field}

In this section we will use part of the BPS equations (2.67) and (2.68) to eliminate the terms quadratic in derivatives, to find equations which are linear in derivative terms. These can then be used to solve for the remaining component of the gauge field, $A_{\beta_{2}}$. Eqs. (2.67a)$(2.67 \mathrm{c})$ can be regarded as a set of linear equations for $\left(B_{1}, B_{2}, B_{3}\right)$ and we can solve them for the terms quadratic in derivatives. This yields

$$
\begin{aligned}
\sinh \rho B_{1}= & \cos \theta\left[\lambda \sec ^{2} z \cosh ^{2} \rho \partial_{x} \rho+\cos ^{2} \theta \partial_{z} \theta+\frac{F_{z}}{1-x^{2}} C_{+}\right] \\
x \sec ^{2} \theta B_{2}= & \operatorname{coth} \rho \tan z \sec ^{2} z\left(A_{1}+A_{2} \tan \theta\right)+\lambda A_{3} \sin \theta+\lambda \operatorname{csch} \rho \tan z \partial_{z} \sin \theta \\
& +F_{z} \tanh \rho+\frac{\lambda F_{z}}{1-x^{2}} \sec \theta \operatorname{csch} \rho \tan z C_{+}, \\
x \sec \theta B_{3}= & \cosh \rho\left(x \csc z \sec z\left(A_{2}-\tan \theta A_{1}\right)+A_{3} \sin \theta \cot z\right. \\
& \left.+\lambda \operatorname{coth} \rho \sec ^{2} z\left(A_{1}+A_{2} \tan \theta\right)\right) \\
& -\lambda \operatorname{csch} \rho \cot z C_{-} \partial_{z} \theta+\frac{\lambda F_{z}}{1-x^{2}}(\sinh \rho \cot z+\cosh \rho \operatorname{coth} \rho \tan z),
\end{aligned}
$$

where

$$
C_{ \pm}=x \sinh \rho \sin \theta \pm \lambda \tan z \cos \theta \cosh \rho .
$$

We will not use (2.68a) to avoid introducing square roots, and using the solution for $\left(B_{1}, B_{2}, B_{3}\right)$ thus leaves us with four equations which are linear in derivative terms, namely (2.67d) and (2.68b)-(2.68d). A linear combination which is particularly helpful can 
be isolated as follows. We solve (2.67d) and (2.68b), with $B_{i}$ replaced according to (2.69), for $\partial_{x} \rho$ and $\partial_{x} \theta$, and use the result in (2.68c) and (2.68d). They then both become

$$
\partial_{z}(x \sinh \rho \cos \theta-\lambda \tan z \cosh \rho \sin \theta)+F_{z}=0 .
$$

This equation can be integrated straightforwardly for the gauge potential, which has to be given by the expression in the round brackets up to a function of $x$ only. We can now use this result for $F_{z}$ in eq. (2.67d), solve for $\partial_{z} \theta$ and use the result in (2.68b). This yields

$$
\partial_{x}(x \sinh \rho \cos \theta-\lambda \tan z \cosh \rho \sin \theta)+F_{x}=0 .
$$

Together with (2.71) this fixes the solution for the gauge field to

$$
A_{\beta_{2}}=\lambda \tan z \cosh \rho \sin \theta-x \sinh \rho \cos \theta+\mathcal{A}_{0},
$$

with an arbitrary constant $\mathcal{A}_{0}$. The Bianchi identity for $F$ is then automatically satisfied. We will derive one more useful quasilinear equation before collecting and discussing the complete set of remaining equations in the next subsection. To this end, we use the solution for the gauge field, solve eq. (2.67d) for $\partial_{z} \theta$ and use the result in (2.68c). The resulting equation is

$$
\left(1-x^{2}\right) \cosh \rho\left(\lambda x \partial_{x} \theta+\tan z \partial_{x} \rho-\lambda \sin \theta \cos \theta\right)+\cos \theta F_{z}=0 .
$$

\subsection{The remaining equations}

We will now collect and discuss the complete set of remaining equations after solving for the gauge field. Upon using the solution for the gauge field (2.73) and the quasilinear equation (2.74), the $\mathcal{Q}_{\mathcal{K}}=0$ equations (2.67a) $-(2.67 \mathrm{~d})$ and the last three of the $\mathcal{Q}_{\mathbb{1}}-h \mathbb{1}=0$ equations (2.68b)-(2.68d) all become equivalent. This may be verified by solving (2.74) for $\partial_{x} \theta$ and replacing it everywhere, after using (2.73). This leaves only the first of the $\mathcal{Q}_{\mathbb{1}}-h \mathbb{1}=0$ equations, (2.68a), and (2.74) in addition. We are thus left with three equations for two functions $\rho, \theta$, which we repeat for convenience. Of the now equivalent eqs. (2.67a)-(2.67d) and (2.68b)-(2.68d), we pick (2.67d), leaving us with

$$
\begin{aligned}
\left(1-x^{2}\right) \cosh \rho\left(\lambda x \partial_{x} \theta+\tan z \partial_{x} \rho-\lambda \sin \theta \cos \theta\right)+\cos \theta F_{z} & =0, \\
\cos ^{2} \theta\left(\cos \theta A_{3}-\lambda \tan \theta \tanh \rho F_{z}\right)-\tanh \rho\left(\lambda x B_{1}+\tan z B_{2}\right)-\sec ^{2} z F_{x} & =0, \\
-\frac{h}{\sqrt{1-x^{2}}}+\cos \theta \cosh ^{2} \rho\left[\cos ^{2} \theta-\lambda \tanh \rho \tan z\left(\left(1-x^{2}\right) \partial_{x} \theta+\frac{x}{2} \sin 2 \theta\right)\right] & \\
+\frac{\lambda}{2} \sin 2 \theta\left(x \cos \theta \partial_{z} \rho+\cosh \rho F_{z}\right)+\lambda x \cosh \rho B_{1}+\lambda\left(1-x^{2}\right) \cos \theta B_{3} & =0 .
\end{aligned}
$$

These three equations imply all the others and the Bianchi identity. We will now show that the third equation, (2.77), is actually also implied already by (2.75) and (2.76). Using the shorthands $B_{i}$ and the definition of $h$, we can write $h^{2}$ as

$$
\begin{aligned}
\frac{h^{2}}{1-x^{2}}= & \cos ^{4} \theta \cosh ^{2} \rho+\cos ^{2} z\left[B_{1}^{2}+B_{2}^{2}+\left(1-x^{2}\right) \cos ^{2} \theta B_{3}^{2}+\left(\left(\partial_{z} \theta\right)^{2}+\left(\partial_{z} \rho\right)^{2}\right) \cos ^{4} \theta\right] \\
& +\left(1-x^{2}\right)\left[\left(\partial_{x} \theta\right)^{2}+\left(\partial_{x} \rho\right)^{2}\right] \cos ^{2} \theta \cosh ^{2} \rho+F_{x}^{2} \cosh ^{2} \rho+\frac{F_{z}^{2} \cos ^{2} \theta \cos ^{2} z}{1-x^{2}} .
\end{aligned}
$$


We now isolate the term involving $h$ in (2.77) and square the equation afterwards, to eliminate the square root. This results in

$$
\begin{aligned}
\frac{h^{2}}{1-x^{2}}= & {\left[\cos \theta \cosh ^{2} \rho\left[\cos ^{2} \theta-\lambda \tanh \rho \tan z\left(\left(1-x^{2}\right) \partial_{x} \theta+\frac{x}{2} \sin 2 \theta\right)\right]\right.} \\
& \left.+\frac{\lambda}{2} \sin 2 \theta\left(x \cos \theta \partial_{z} \rho+\cosh \rho F_{z}\right)+\lambda x \cosh \rho B_{1}+\lambda\left(1-x^{2}\right) \cos \theta B_{3}\right]^{2} .
\end{aligned}
$$

With the expression for $h^{2}$ in (2.78) we see that this equation is quadratic in the $B_{i}$, and thus quartic in derivative terms. However, using (2.69) reduces it to an equation which is only quadratic in derivative terms. To further process it, we solve (2.75) for $\partial_{x} \theta$ and eliminate it in (2.76) and in (2.79). Eq. (2.76) is quadratic in derivative terms, and after eliminating $\partial_{x} \theta$ via $(2.75)$ it contains a $\left(\partial_{z} \rho\right)\left(\partial_{z} \theta\right)$ term. We solve $(2.76)$ for $\left(\partial_{z} \rho\right)\left(\partial_{z} \theta\right)$ and use the result to eliminate that particular quadratic derivative term in (2.79) with the $B_{i}$ replaced according to (2.69). After this step (2.79) collapses to zero, showing that the equation is implied by (2.75) and (2.76).

The remaining equations are therefore only (2.75) and (2.76). These two equations for slipping and bending mode, together with the solution for the gauge field (2.73), are equivalent to the entire set of equations in (2.67) and (2.68). We rewrite them as follows. With

$$
G_{\mathfrak{a}}=\lambda \sinh \rho \sin ^{2} \theta \partial_{\mathfrak{a}}(x \cot \theta)-\partial_{\mathfrak{a}}(\tan z \cosh \rho),
$$

they become

$$
\begin{aligned}
-\left(1-x^{2}\right) \cosh \rho G_{x}+\sinh \rho \cos \theta F_{z} & =0, \\
\sinh \rho\left(G_{z} F_{x}-G_{x} F_{z}\right)-\cos ^{3} \theta G_{z}-\sec ^{2} z \cosh ^{3} \rho \cos ^{3} \theta & =0 .
\end{aligned}
$$

We have not been able to find a closed-form solution to these equations. But to validate that the equations are correct, we solved them to cubic order in a perturbative expansion around the solution where $\rho$ and $\theta$ vanish identically, and verified that these perturbative solutions solve the equations of motion resulting from the DBI action in (2.2). Since the cubic order in the perturbative expansion is sensitive to all terms in the equations, this provides a strong consistency check on the full non-linear equations. We suspect that this check can be extended to more generally show that the non-linear BPS equations imply the equations of motion, but will leave this question open here. We will study physical applications of the perturbative solutions in more detail in the next sections.

\section{Perturbative solutions for $\mathrm{AdS}_{3}$ defects}

In this section we study perturbative solutions to the BPS equations derived in the previous section. We start with the general linearized solution, which describes infinitesimal fluctuations around the massless embedding where $\rho, \theta$ and $A_{\beta_{2}}$ vanish identically. We will find a large space of supersymmetric 'vacuum states', where the expectation values of all the supersymmetric operators sourced by combinations of slipping mode, bending mode and 
gauge field can be varied continuously and independently on one of the $\mathrm{AdS}_{3}$ geometries obtained as $r \rightarrow \pm \infty$. Once the expectation values are fixed on one of the $\mathrm{AdS}_{3}$ patches, they are then fixed on the remaining one as well. We will close with a qualitative discussion of higher orders in the perturbative expansion and of implications of that general structure for non-linear solutions.

To make the expansion explicit we introduce a small parameter $\kappa$ and expand around the massless embedding in the form

$$
\rho=\sum_{n} \kappa^{n} \rho^{(n)}, \quad \theta=\sum_{n} \kappa^{n} \theta^{(n)} .
$$

The solution for the gauge field (2.73) can be expanded in a similar way and at linear order becomes

$$
A_{\beta_{2}}^{(1)}=\lambda \tan z \theta^{(1)}-x \rho^{(1)}+\mathcal{A}_{0}^{(1)} .
$$

With only $A_{\beta_{2}}$ non-vanishing, we automatically have $d_{\mathrm{S}^{2}}^{\dagger} A=0$, or $\nabla^{i} A_{i}=0$ with $i$ running over the $\mathrm{S}^{2}$ indices corresponding to $\left(\beta_{1}, \beta_{2}\right)$ only. The linearized versions of $(2.81)$ read

$$
\begin{aligned}
\partial_{z} A_{\beta_{2}}^{(1)}+\left(1-x^{2}\right) \partial_{x}\left(\lambda x \theta^{(1)}+\tan z \rho^{(1)}\right)-2 \lambda\left(1-x^{2}\right) \theta^{(1)} & =0, \\
\cos ^{2} z \partial_{z}\left(\lambda x \theta^{(1)}+\tan z \rho^{(1)}\right)-\partial_{x} A_{\beta_{2}}^{(1)}-2 \rho^{(1)} & =0 .
\end{aligned}
$$

We change variables and define

$$
\phi=\frac{\sec ^{2} z}{x^{2}+\tan ^{2} z} A_{\beta_{2}}^{(1)}, \quad \zeta=\frac{\lambda x \theta^{(1)}+\tan z \rho^{(1)}}{x^{2}+\tan ^{2} z} .
$$

Eqs. (3.3) then become

$$
\begin{aligned}
\cos ^{2} z \partial_{z} \phi+\left(1-x^{2}\right) \partial_{x} \zeta & =0 \\
\partial_{z} \zeta-\partial_{x} \phi & =0
\end{aligned}
$$

Taking a derivative of the first equation with respect to $x$ and then using the result in the second equation leaves us with an equation for $\zeta$ alone,

$$
\cos ^{2} z \partial_{z}^{2} \zeta+\partial_{x}\left(1-x^{2}\right) \partial_{x} \zeta=0
$$

This equation can be solved by separation of variables. We write $\zeta=p_{\ell}(x) \zeta_{\ell}(z)$ and conveniently introduce a constant $\ell$ such that the above equation implies

$$
\partial_{x}\left(1-x^{2}\right) \partial_{x} p=-\ell(\ell+1) p, \quad \cos ^{2} z \partial_{z}^{2} \zeta_{\ell}=\ell(\ell+1) \zeta_{\ell} .
$$

The $x$-dependent part is the Legendre equation, and the requirement of regularity at $x=1$ and $x=0$, corresponding to $\beta_{1}=0$ and $\beta_{1}=\pi$, respectively, forces us to choose the Legendre functions of the first kind, $P_{\ell}$, with $\ell$ an integer. ${ }^{5}$ Since $P_{\ell}=P_{-\ell-1}$, we can

\footnotetext{
${ }^{5}$ Note that regularity of $\theta$ and $\rho$ implies regularity of $\zeta, \phi$ for $z \in\left(0, \frac{\pi}{2}\right)$.
} 
restrict to non-negative $\ell$ w.o.l.g. The result should not come as a surprise, as the $P_{\ell}$ are the polynomials appearing in the spherical harmonics $Y_{\ell, 0}$. Summing up, we have

$$
\zeta=\sum_{\ell=0}^{\infty} P_{\ell}(x) \zeta_{\ell}(z), \quad \cos ^{2} z \partial_{z}^{2} \zeta_{\ell}=\ell(\ell+1) \zeta_{\ell}
$$

Solving for $\zeta_{\ell}$ yields

$$
\zeta_{\ell}=c_{\ell, 1} f_{\ell}+c_{\ell, 2} f_{-\ell-1}, \quad f_{\ell}=(\cos z)^{\ell+1}{ }_{2} F_{1}\left(\frac{\ell+1}{2}, \frac{\ell+1}{2}, \ell+\frac{3}{2}, \cos ^{2} z\right) .
$$

Note that $\ell(\ell+1)$ is invariant under $\ell \rightarrow-\ell-1$, but $f_{\ell}$ is not. The remaining task is to solve for $\phi$. We note that $\phi$ is defined in terms of the one form component $A_{\beta_{1}}$ by a simple rescaling. In particular, it has to satisfy the same regularity conditions, namely vanish at $x=0$ and $x=1$, corresponding to $\beta_{1}=\pi$ and $\beta_{1}=0$, respectively. We should therefore expand it in 1-form spherical harmonics. Just like $A_{\beta_{2}}$, $\phi$ is part of a divergencefree vector field, and this fixes the kind of vector spherical harmonics that appear. To make the normalization convention explicit, we take

$$
\vec{Y}_{\ell, 0}=\star_{\mathrm{S}^{2}} d P_{\ell}=\left(1-x^{2}\right) \frac{d}{d x} P_{\ell}(x)
$$

and expand

$$
\phi=\sum_{\ell=0}^{\infty} \phi_{\ell}(z) \vec{Y}_{\ell, 0} .
$$

Note that the $\ell=0$ term vanishes regardless of the value of $\phi_{0}$. Using this expansion in the second equation of (3.5) shows that

$$
\zeta_{0}^{\prime}=0, \quad \phi_{\ell}=-\frac{1}{\ell(\ell+1)} \zeta_{\ell}^{\prime}, \quad \ell \geq 1 .
$$

$\zeta_{0}^{\prime}$ is indeed a constant and the first equation fixes $c_{0,1}=0$. It will be convenient to recast the expansion of $\phi$ in one-form spherical harmonics as expansion in the Legendre polynomials themselves. Using $\left(1-x^{2}\right) \frac{d}{d x} P_{\ell}=-\ell\left(x P_{\ell}-P_{\ell-1}\right)$ and $(2 \ell+1) x P_{\ell}=(\ell+$ 1) $P_{\ell}+\ell P_{\ell-1}$, we find

$$
\left(1-x^{2}\right) \frac{d}{d x} P_{\ell}=-\frac{\ell(\ell+1)}{2 \ell+1}\left(P_{\ell+1}-P_{\ell-1}\right) .
$$

With the understanding that $\zeta_{-1}^{\prime}=\zeta_{0}^{\prime}=0$, the expansion for $\phi$ becomes

$$
\phi=\sum_{\ell=0}^{\infty} P_{\ell}(x) \varphi_{\ell}(z), \quad \varphi_{\ell}=\frac{\zeta_{\ell-1}^{\prime}}{2 l-1}-\frac{\zeta_{\ell+1}^{\prime}}{2 l+3} .
$$

To translate back to $\theta^{(1)}$ and $\rho^{(1)}$ we use (3.4), which can be solved to give

$$
\theta^{(1)}=\lambda(\sin z \cos z \phi+x \zeta), \quad \rho^{(1)}=\tan z \zeta-x \cos ^{2} z \phi .
$$


This yields

$$
\begin{array}{ll}
\theta^{(1)}=\sum_{\ell=0}^{\infty} P_{\ell}(x) \theta_{\ell}^{(1)}(z) & \theta_{\ell}^{(1)}=\lambda\left[\sin z \cos z \varphi_{\ell}+\frac{\ell \zeta_{\ell-1}}{2 \ell-1}+\frac{(\ell+1) \zeta_{\ell+1}}{2 \ell+3}\right], \\
\rho^{(1)}=\sum_{\ell=0}^{\infty} P_{\ell}(x) \rho_{\ell}^{(1)}(z) & \rho_{\ell}^{(1)}=\tan z \zeta_{\ell}-\cos ^{2} z\left[\frac{(\ell+1) \varphi_{\ell+1}}{2 \ell+3}+\frac{\ell \varphi_{\ell-1}}{2 \ell-1}\right] .
\end{array}
$$

Finally, the solution for the gauge field (3.2) with (3.4) becomes

$$
A_{\beta_{2}}^{(1)}=-\left(1-x^{2}\right) \cos ^{2} z \phi+\phi+\mathcal{A}_{0}^{(1)} .
$$

Simply substituting the expressions for $\rho^{(1)}$ and $\theta^{(1)}$ would yield an expansion in scalar spherical harmonics, which would be straightforward and possible but make regularity obscure. The more natural expansion is again in terms of the 1-form spherical harmonics (3.10). The second term can be brought into this form directly, using (3.11). The first term can be rearranged using the expansion (3.14) and $(2 n+1) P_{n}=\frac{d}{d x}\left(P_{n+1}-P_{n-1}\right)$. The result is

$$
A_{\beta_{2}}^{(1)}=\sum_{\ell=1}^{\infty} A_{\ell}^{(1)}(z)\left(1-x^{2}\right) \frac{d}{d x} P_{\ell}(x), \quad A_{\ell}^{(1)}=\cos ^{2} z\left[\frac{\phi_{\ell+1}}{2 \ell+3}-\frac{\phi_{\ell-1}}{2 \ell-1}\right]-\frac{\zeta_{\ell}^{\prime}}{\ell(\ell+1)},
$$

where $\mathcal{A}_{0}^{(1)}$ has been fixed to ensure regularity at the poles of $\mathrm{S}^{2}$. All functions appearing as radial profiles are hypergeometric functions with $\operatorname{argument} \cos ^{2} z$. They have the usual regular singular points for $\cos ^{2} z=0$ and $\cos ^{2} z=1$ and are regular for $\cos ^{2} z \in(0,1)$. Geometrically, $\cos ^{2} z=0$ corresponds to the conformal boundaries at $r \rightarrow \pm \infty$, while $\cos ^{2} z=1$ corresponds to $r=0$ where we need regular solutions. For any choice of the constants the solutions are all regular at $z=0$, and there are therefore no constraints from regularity in the interior of $\mathrm{AdS}_{5}$.

With the general linearized solution in hand we can now analyze the near-boundary behavior from which the one-point functions can be deduced straightforwardly. The D5branes intersect the conformal boundary at $r \rightarrow \pm \infty$, corresponding to $z \rightarrow \pm \frac{\pi}{2}$, and each of these limits corresponds to an $\mathrm{AdS}_{3}$ defect. To analyze the near-boundary behavior of the solutions we switch to Fefferman-Graham coordinates $r=\mp \log (u / 2)$ or $z= \pm\left(2 \tan ^{-1}\left(\frac{2}{u}\right)-\right.$ $\left.\frac{\pi}{2}\right)$. This transforms the $\mathrm{AdS}_{4}$ metric in (2.1) to Fefferman-Graham gauge, where the terms in the near-boundary expansion of the bulk fields can be related directly to the sources and vacuum expectation values in the CFT. We then find

$$
\begin{gathered}
\theta_{\ell}^{(1)}=\lambda u^{1-\ell}\left(d_{\ell, 2}+\ldots\right)+\lambda u^{2+\ell}\left(d_{\ell, 1}+\ldots\right), \\
\pm \frac{\rho_{\ell}^{(1)}-\ell \mathcal{A}_{\ell}^{(1)}}{2 \ell+1}=\left(\frac{d_{\ell+1,2}}{\ell+1}+\ldots\right) u^{-\ell-1}-\left(\frac{\ell+3}{(2 \ell+3)(2 \ell+5)} d_{\ell+1,1}+\ldots\right) u^{4+\ell}, \\
\pm \frac{\rho_{\ell}^{(1)}+(\ell+1) \mathcal{A}_{\ell}^{(1)}}{2 \ell+1}=\left(\frac{2-\ell}{(2 \ell-3)(2 \ell-1)} d_{\ell-1,2}+\ldots\right) u^{3-\ell}+\left(\frac{d_{\ell-1,1}}{\ell}+\ldots\right) u^{\ell}, \quad
\end{gathered}
$$


where the upper/lower choice of the sign in the last two lines corresponds to the behavior at the $\mathrm{AdS}_{3}$ obtained as $r \rightarrow+\infty / r \rightarrow-\infty$. The dots denote terms which vanish at $u=0$ and we have redefined the coefficients as

$$
d_{\ell, 1}=\frac{\ell(\ell+1)}{4 \ell^{2}-1} c_{\ell-1,1}+c_{\ell+1,1}, \quad d_{\ell, 2}=c_{\ell-1,2}+\frac{\ell(\ell+1) c_{\ell+1,2}}{(2 \ell+1)(2 \ell+3)} .
$$

The leading terms in the expansions (3.20) show that the slipping mode corresponds to an operator with $\Delta_{+}=2+\ell$ and $\Delta_{-}=1-\ell$. The two linear combinations of bending mode and gauge field source one operator with $\Delta_{+}=\ell+4$ and $\Delta_{-}=-1-\ell$, and another one with $\Delta_{+}=\ell$ and $\Delta_{-}=3-\ell$. In standard quantization the $d_{\ell, 2}$ are the sources and the $d_{\ell, 1}$ parametrize the expectation values. For the low-lying operators the roles are exchanged if alternative quantization is chosen. As discussed in detail for the D3/D5 system describing a flat defect in [17], supersymmetry fixes this choice and requires alternative quantization for the low-lying $\ell=1$ mode in the coupled sector. The scaling dimensions match those found for a flat defect in [17].

At the linearized level each perturbation can be turned on independently, and as discussed above there are no further regularity conditions from the interior of the bulk AdS. So the $d_{\ell, 1}$ and $d_{\ell, 2}$ can be chosen independently, and we can in particular set all sources to zero and still dial the subleading terms in the asymptotic expansions (3.20). With all sources vanishing there are no extra contributions from holographic renormalization and the subleading terms directly correspond to the one-point functions. The expectation values of the operators sourced by slipping mode, bending mode and gauge field are linked, since they are all three proportional to just one set of constants given by the $d_{\ell, 1}$. This of is a result of supersymmetry. But we nevertheless find a large "moduli space" of supersymmetric vacuum states, where the one-point functions can be chosen on one of the $\mathrm{AdS}_{3}$ making up the defect and are then fixed on the remaining $\mathrm{AdS}_{3}$. We can make this very explicit for the special case of a pure mass deformation, where the slipping mode only has a non-vanishing $\theta_{0}$ mode and the bending mode only has a non-vanishing $\rho_{1}$ mode. The explicit solution is given by

$$
\theta^{(1)}=P_{0}(x) \cos z(m \sin z+c \cos z), \quad \rho^{(1)}=\lambda P_{1}(x) \cos z(c \sin z-m \cos z),
$$

where $m$ parametrizes the mass deformation and $c$ the chiral condensate. Both can be varied independently, so the expectation value on one of the $\mathrm{AdS}_{3}$ parts of the defect is not fixed in terms of the source on that part. But sources and expectation values are related between the two $\mathrm{AdS}_{3}$ parts obtained as $z \rightarrow \pm \frac{\pi}{2}$.

We will close this section with qualitative comments on the higher orders in the perturbative expansion. Starting from the linearized solution corresponding to a pure mass deformation in (3.22), it is straightforward to then expand and solve the BPS equations to higher orders in the fluctuations. The linear-order fluctuations do not source the fields at quadratic order, and it is consistent to set them to zero

$$
\theta^{(2)}=\rho^{(2)}=0 \text {. }
$$

At cubic order in the mass deformation, the linear-order fluctuations $\theta_{0}^{(1)}$ and $\rho_{1}^{(1)}$ do appear as sources for $\theta_{0}^{(3)}$ and $\rho_{1}^{(3)}$ and those modes are non-vanishing. Moreover, they also trigger 
higher spherical harmonics in $\theta$ and $\rho$. Namely, $\theta_{0}^{(1)}$ and $\rho_{1}^{(1)}$ appear as sources in the equations for $\theta_{2}^{(3)}$ and $\rho_{3}^{(3)}$, and consistent solutions consequently require non-vanishing $\theta_{2}$ and $\rho_{3}$ as well. At cubic order the solution therefore takes the form

$$
\theta^{(3)}=\theta_{0}^{(3)}(z) P_{0}(x)+\theta_{2}^{(3)}(z) P_{2}(x), \quad \rho^{(3)}=\rho_{1}^{(3)}(z) P_{1}(x)+\rho_{3}^{(3)}(z) P_{3}(x) .
$$

The general solution for each of the higher modes $\theta_{0}^{(3)}, \theta_{2}^{(3)}$ and $\rho_{1}^{(3)}, \rho_{3}^{(3)}$ is a linear combination of a particular solution to the inhomogeneous equation with the general solution to the homogeneous equation. This introduces four additional constants and the general solution is rather bulky. However, physically we still want to describe a pure mass deformation, which means the terms in the near-boundary expansions of $\theta_{2}^{(3)}$ and $\rho_{3}^{(3)}$ that correspond to sources in the CFT should be zero - on both $\mathrm{AdS}_{3}$ parts that make up the defect. This fixes two of the four constants, and afterwards the solutions for $\theta_{2}^{(3)}$ and $\rho_{3}^{(3)}$ take a very simple form, namely

$$
\theta_{2}^{(3)}=-\frac{1}{3} \theta_{0}^{(1)}\left(\rho_{1}^{(1)}\right)^{2}, \quad \rho_{3}^{(3)}=\frac{1}{15}\left(\rho_{1}^{(1)}\right)^{3} .
$$

Expanding these modes near the conformal boundaries at $z \rightarrow \pm \frac{\pi}{2}$ shows that the terms corresponding to the expectation value of the dual operator in $\theta_{2}^{(3)}$ are non-zero, and the same applies for the two combinations of $\rho_{3}^{(3)}$ and $A_{3}^{(3)}$. The remaining two constants appear in $\rho_{1}^{(3)}$ and $\theta_{0}^{(3)}$ only and they can be fixed by demanding that the mass and expectation values for the lowest spherical harmonics are not redefined at cubic order. We will not give the explicit form for $\rho_{1}^{(3)}$ and $\theta_{0}^{(3)}$ here, and leave a more detailed study of the non-linear equations for the future. At the technical level, the need for higher spherical harmonics implies that a separation ansatz can not be used to solve the BPS equations, at least not in these variables. But the simple form of the higher modes in (3.25) certainly suggests that a separation of variables ansatz may work after suitably chosen field redefinitions.

\section{$4 \quad \mathrm{~S}^{3}$ defects and $\mathrm{S}^{4}$ partition function from holography}

In this section we analytically continue the embeddings for the $\mathrm{AdS}_{4}$ slicing to global Euclidean $\mathrm{AdS}_{5}$, where the slices are $\mathrm{S}^{4}$. The role of IR regularity conditions is qualitatively different in that case and fixing the CFT sources fixes a unique vacuum state and a unique D5-brane embedding. We study the perturbative embeddings for a pure mass deformation and compute the partition function for $\mathcal{N}=4 \mathrm{SYM}$ on $\mathrm{S}^{4}$ with massive defects on an equatorial $\mathrm{S}^{3}$ holographically, to quadratic order in the mass parameter.

To get from the Lorentzian AdS slicing to Euclidean spheres, we use the following strategy. Firstly, the equations (2.81) do not depend on the chosen coordinates on the $\mathrm{AdS}_{3}$ slices. So we can straightforwardly implement the analytic continuation

$$
r=\hat{r}+\frac{i \pi}{2}, \quad g_{\mathrm{AdS}_{3}} \rightarrow-g_{\mathrm{S}^{3}} .
$$

The continuation from $\mathrm{AdS}_{3}$ to $\mathrm{S}^{3}$ can proceed via the continuation from $\mathrm{AdS}_{3}$ to $\mathrm{dS}_{3}$, as used in [14], and from there to $\mathrm{S}^{3}$. Note that this results in an $\mathrm{S}^{3}$ with negative signature. 
This turns the $\mathrm{AdS}_{5}$ part of the metric (2.1) into

$$
g_{\mathrm{AdS}_{5}}=d \rho^{2}+\cosh ^{2} \rho\left[d \hat{r}^{2}+\sinh ^{2} \hat{r} g_{\mathrm{S}^{3}}\right],
$$

which is Euclidean $\mathrm{AdS}_{5}$, or $\mathbb{H}_{5}$, sliced by $\mathbb{H}_{4}$ surfaces. The massless embedding $\rho(r) \equiv$ $\rho(\hat{r}) \equiv 0$ now describes a D5 wrapping $\mathbb{H}_{4} \times \mathrm{S}^{2}$, and the deformations described by $(2.81)$ with $r \rightarrow \hat{r}$ generally preserve the $\mathrm{S}^{3}$ isometries.

We now implement a coordinate transformation such that the metric in (4.2) turns into

$$
g_{\mathrm{AdS}_{5}}=d R^{2}+\sinh ^{2} R\left[d \chi^{2}+\cos ^{2} \chi g_{\mathrm{S}^{3}}\right],
$$

where the terms in square brackets combine to an $\mathrm{S}^{4}$. This can be achieved by setting

$$
\cosh R=\cosh \rho \cosh \hat{r}, \quad \sinh R \sin \chi=\sinh \rho .
$$

The embedding $\rho \equiv 0$ translates to $\chi \equiv 0$, which wraps an equatorial $\mathrm{S}^{3}$ inside $\mathrm{S}^{4}$, as desired. As Euclidean DBI action for the D5-branes we have

$$
S_{\mathrm{D} 5}=T_{5} \int_{\Sigma_{6}} d^{6} \xi \sqrt{\operatorname{det}(g+F)}+T_{5} \int_{\Sigma_{6}} C_{4} \wedge F
$$

with $C_{4}$ of the background solution given by

$$
C_{4}=\zeta(\chi) \sinh ^{4} R \sin ^{2} \alpha_{1} \sin \alpha_{2} d R \wedge d \alpha_{1} \wedge d \alpha_{2} \wedge d \alpha_{3}+\ldots, \quad \zeta^{\prime}(\chi)=-4 \cos ^{3} \chi .
$$

Note the sign in $\zeta^{\prime}$ to account for the ordering of $d R$ and $d \chi$ in $d C_{4}$, to get the positive volume form.

The BPS equations can be obtained by analytically continuing and coordinate transforming those for the $\mathrm{AdS}_{4}$ slicing, and we can readily obtain the solution for the gauge field by that procedure from the solution for the $\mathrm{AdS}_{4}$ slicing in (2.73). This yields

$$
A_{\beta_{2}}=i \lambda \sin \theta \cosh R-x \cos \theta \sinh R \sin \chi+\mathcal{A}_{0} .
$$

\subsection{Continuing perturbative solutions}

We now want to use (4.4) to obtain perturbative embeddings for the sphere slicing. For an arbitrary given embedding in the $\mathrm{AdS}_{4}$ slicing coordinates, described by $\rho, \theta, A_{\beta_{2}}$ as functions of $r$ and $\beta_{1}$, we first implement (4.1) and replace $r$ by $\hat{r}$. We then use the perturbative expansion as set up in eq. (3.1) and solve eq. (4.4) for $\hat{r}$ in terms of $R$ in an expansion in $\kappa$, and likewise for $\chi$ in terms of $\rho$. At linear order in the fluctuations this yields

$$
r(R)=R+\frac{i \pi}{2}+\mathcal{O}\left(\kappa^{2}\right), \quad \sinh R \chi^{(1)}(R)=\rho^{(1)}\left(r^{(0)}(R)\right) .
$$

To find the solution for the embedding in the $\mathrm{S}^{4}$ slicing, we analytically continue the equation, rather than the solution. The linearized BPS equations for the $\mathrm{S}^{4}$ slicing with the analytic continuation derived above are given by (3.3) with $2 \tanh ^{-1} \tan (z / 2)=r^{(0)}(R)$ and $\rho^{(1)}=-i \sec z \chi^{(1)}$. The solution corresponding to a mass deformation is

$$
\theta^{(1)}=\operatorname{csch}^{2} R(m \cosh R+c), \quad \chi^{(1)}=i \lambda \cos \beta_{1} \operatorname{csch}^{3} R(m+c \cosh R) .
$$


For the gauge field the solution is obtained from (4.7), and the constant $\mathcal{A}_{0}$ has to be fixed such that $A_{\beta_{2}}^{(1)}$ vanishes at $\beta_{1}=0$ and $\beta_{1}=\pi$, to get a regular one form on $\mathrm{S}^{2}$. This yields $\mathcal{A}_{0}=-i \kappa \lambda m$ and consequently

$$
A_{\beta_{2}}^{(1)}=i \lambda \sin ^{2} \beta_{1} \operatorname{csch}^{2} R(c \cosh R+m) .
$$

Note that the gauge field and bending mode are imaginary. For the gauge field this is parallel to the $\mathrm{S}^{4}$ slicing solutions for D7-branes obtained in [14], and for the bending mode it naturally aligns with its mixing with the gauge field. The combined solution to the BPS equations in (4.9) indeed solves the equations of motion derived from the DBI action (4.5), (4.6).

The next step is to implement regularity conditions at $R=0$. The expansion of the slipping and bending modes reads

$$
\theta^{(1)}=\frac{c+m}{R^{2}}+\mathcal{O}(1), \quad \sec \beta_{1} \chi^{(1)}=\frac{i \lambda(c+m)}{R^{3}}-\frac{i \lambda m}{2 R}+\mathcal{O}(R) .
$$

The slipping mode can be rendered finite at the origin by setting

$$
c=-m
$$

This leaves an $R^{-1}$ divergence in the bending mode. However, the relevant quantity that needs to be finite is $\sinh R \chi$ and $\operatorname{since} \sinh R$ vanishes as $R \rightarrow 0$ this combination is indeed finite. Eventually the bending mode mixes with the gauge field and we will see below that the decoupled modes are indeed regular, so this completes the discussion of the regularity conditions.

\subsection{Holographic one-point functions}

In this section we compute the one-point functions for the operators sourced by the perturbations to the massless embedding discussed in the previous section. The fluctuations satisfy a coupled set of equations of motion and we first need to reformulate the action in terms of linear combinations of $\chi, \theta$ and $A_{\beta_{2}}$ such that the equations of motion decouple. It is these linear combinations that have a consistent near-boundary behavior with two generalized boundary values corresponding to source and expectation value for a dual operator. We again work perturbatively and expand the action as

$$
S_{\mathrm{D} 5}=\sum_{n} \kappa^{n} S_{\mathrm{D} 5}^{(n)}
$$

The $\mathcal{O}\left(\kappa^{0}\right)$ part is independent of the fluctuations but will nevertheless be useful for the computation of the partition function. It is given by

$$
S_{\mathrm{D} 5}^{(0)}=T_{5} V_{\mathrm{S}^{2}} V_{\mathrm{S}^{3}} \int d R \sinh ^{3} R
$$


The interesting part for the fluctuations is the $\mathcal{O}\left(\kappa^{2}\right)$ term, which is given by

$$
\begin{aligned}
\frac{2 S_{\mathrm{D} 5}^{(2)}}{V_{\mathrm{S}^{1}} V_{\mathrm{S}^{3}} T_{5}}= & \int d R d \beta_{1} \sin \beta_{1} \sinh ^{3} R\left[\left(\partial_{\beta_{1}} \theta^{(1)}\right)^{2}+\left(\partial_{R} \theta^{(1)}\right)^{2}-2\left(\theta^{(1)}\right)^{2}\right. \\
& +\sinh ^{2} R\left(\left(\partial_{\beta_{1}} \chi^{(1)}\right)^{2}+\left(\partial_{R} \chi^{(1)}\right)^{2}\right)-3\left(\chi^{(1)}\right)^{2} \\
& \left.+\frac{\left(\partial_{\beta_{1}} A_{\beta_{2}}^{(1)}\right)^{2}+\left(\partial_{R} A_{\beta_{2}}^{(1)}\right)^{2}}{\sin ^{2} \beta_{1}}-\frac{8 \sinh R}{\sin \beta_{1}} \chi^{(1)} \partial_{\beta_{1}} A_{\beta_{2}}^{(1)}\right]
\end{aligned}
$$

Gauge field and bending mode are coupled, and to decouple them we define

$$
\begin{gathered}
\varpi^{(1)}=\csc ^{2} \beta_{1} A_{\beta_{2}}^{(1)}-\sec \beta_{1} \sinh R \chi^{(1)}, \\
\varsigma^{(1)}=2 \csc ^{2} \beta_{1} A_{\beta_{2}}^{(1)}+\sec \beta_{1} \sinh R \chi^{(1)} .
\end{gathered}
$$

The resulting action, using that we have $\ell=1$ modes for $\rho$ and $A_{\beta_{2}}$ and an $\ell=0$ mode for $\theta$, and after integrating over $\beta_{1}$, can be written conveniently as

$$
S_{\mathrm{D} 5}^{(2)}=T_{5} V_{\mathrm{S}^{1}} V_{\mathrm{S}^{3}}\left(S_{\theta^{(1)}}+S_{\varsigma^{(1)}}+S_{\varpi}{ }^{(1)}\right)
$$

where

$$
\begin{aligned}
S_{\theta^{(1)}} & =\int d R \sinh ^{3} R\left(\left(\partial_{R} \theta^{(1)}\right)^{2}-2\left(\theta^{(1)}\right)^{2}\right), \\
S_{\varsigma^{(1)}} & =\int d R \sinh ^{3} R \frac{1}{54}\left[6\left(\partial_{R} \varsigma^{(1)}\right)^{2}-4 \varsigma^{(1)} \operatorname{coth} R \partial_{R} \varsigma^{(1)}+\left(\varsigma^{(1)}\right)^{2} \operatorname{csch}^{2} R(5-9 \cosh (2 R))\right], \\
S_{\varpi(1)} & =\int d R \sinh ^{3} R \frac{2}{27}\left[3\left(\partial_{R} \varpi^{(1)}\right)^{2}-4 \varpi^{(1)} \operatorname{coth} R \partial_{R} \varpi^{(1)}-4\left(\varpi^{(1)}\right)^{2}\left(\operatorname{csch}^{2} R-6\right)\right] .
\end{aligned}
$$

The corresponding equations of motion are

$$
\left(D_{R}^{2}-10\right) \varpi^{(1)}=0, \quad\left(D_{R}^{2}+2\right) \varsigma_{1}^{(1)}=0, \quad\left(D_{R}^{2}+2\right) \theta^{(1)}=0,
$$

where $D_{R}^{2}=\operatorname{csch}^{3} R \partial_{R} \sinh ^{3} R \partial_{R}$. This matches the linearized spectrum for the coupled sector analysis found in [17] for $\ell=1$.

For the solutions in $(4.9)$ the combination $\varpi^{(1)}$ vanishes identically, $\varpi^{(1)} \equiv 0$. For the remaining two fields we will need the near-boundary expansions. Transforming to Fefferman-Graham coordinates by setting $R=-\log (\epsilon / 2)$ and expanding in small $\epsilon$ yields

$$
\theta^{(1)}=m \epsilon+c \epsilon^{2}+\mathcal{O}\left(\epsilon^{3}\right), \quad \varsigma^{(1)}=3 i \lambda c \epsilon+3 i \lambda m \epsilon^{2}+\mathcal{O}\left(\epsilon^{3}\right) .
$$

We note that $\varsigma^{(1)}$ is indeed regular at $R=0$ with the relation between $m$ and $c$ in (4.11). 


\subsubsection{Holographic renormalization}

Since $\varpi^{(1)} \equiv 0$ we only need to discuss the two remaining fields. A crucial point to notice is that, following the discussion in section $3, \varsigma^{(1)}$ is a scalar with alternative quantization, which requires a Legendre transformation in addition to the usual holographic counterterms. Moreover, the action is not in the standard form for a Klein-Gordon field, but differs by total derivatives. So we will discuss the holographic renormalization in some detail. The variation of the action evaluated on shell reads

$$
\begin{aligned}
\delta S_{\theta^{(1)}} & =\left.2 \sinh ^{3} R \delta \theta \partial_{R} \theta\right|_{R \rightarrow \infty}=-\frac{2}{\epsilon} \delta \theta_{0}^{(1)} \theta_{0}^{(1)}-2\left(\theta_{0}^{(1)} \delta \theta_{1}^{(1)}+2 \theta_{1}^{(1)} \delta \theta_{0}^{(1)}\right)+\mathcal{O}(\epsilon), \\
\delta S_{\varsigma^{(1)}} & =\left.\frac{2}{27} \sinh ^{3} R \delta \varsigma^{(1)}\left(3 \partial_{R}-\operatorname{coth} R\right) \varsigma^{(1)}\right|_{R \rightarrow \infty} \\
& =-\frac{8}{27 \epsilon} \varsigma_{0}^{(1)} \delta \varsigma_{0}^{(1)}-\frac{2}{27}\left(4 \varsigma_{0}^{(1)} \delta \varsigma_{1}^{(1)}+7 \varsigma_{1}^{(1)} \delta \varsigma_{0}^{(1)}\right)+\mathcal{O}(\epsilon),
\end{aligned}
$$

For the near-boundary expansions we have used $R=-\log (\epsilon / 2)$ and $\partial_{R}=-\epsilon \partial_{\epsilon}$. Moreover, with a slight abuse of notation, we expanded $\theta^{(1)}=\epsilon \theta_{0}^{(1)}+\epsilon^{2} \theta_{1}^{(1)}+\ldots$ and used an analogous expansion for $\delta \theta^{(1)}$, and likewise for $\varsigma^{(1)}$ and $\delta \varsigma^{(1)}$. We will not use an explicit subscript on $\theta^{(1)}$ to denote the harmonic on $\mathrm{S}^{2}$, such that no confusion should arise. We add the holographic counterterms

$$
\begin{aligned}
& S_{\theta^{(1)}, \mathrm{ct}}=\int d R \sinh ^{3} R\left(\theta^{(1)}\right)^{2}, \\
& S_{\varsigma^{(1)}, \mathrm{ct}}=-\frac{2}{27} \int d R \sinh ^{3} R\left[\left(\varsigma^{(1)}\right)^{2}+3 \varsigma^{(1)} \partial_{R} \varsigma^{(1)}\right] .
\end{aligned}
$$

This produces, as desired, a valid variation for alternative quantization for $\varsigma$. The variations and correspondingly the one-point functions of the CFT operators sourced by $\theta^{(1)}$ and $\varsigma^{(1)}$ are given by

$$
\begin{aligned}
& \delta\left(S_{\theta^{(1)}}+S_{\theta^{(1)}, \mathrm{ct}}\right)=-2 \theta_{1}^{(1)} \delta \theta_{0}^{(1)}=\left\langle\mathcal{O}_{\theta}\right\rangle \delta \theta_{0}^{(1)} \\
& \delta\left(S_{\varsigma^{(1)}}+S_{\varsigma^{(1)}, \mathrm{ct}}\right)=\frac{2}{9} \varsigma_{0}^{(1)} \delta \varsigma_{1}^{(1)}=\left\langle\mathcal{O}_{\varsigma}\right\rangle \delta \varsigma_{1}^{(1)} .
\end{aligned}
$$

We note that the counterterms in (4.21), to quadratic order in the fluctuations, are unique. With the scaling dimensions of the fields one may in principle add finite counterterms like $\left(\theta^{(1)}\right)^{3}$ and $\left(\varsigma^{(1)}\right)^{3}$ with arbitrary coefficients, and such counterterms can indeed be crucial [32]. However. such counterterms would only contribute starting at cubic order in the fluctuations, and do not affect the results derived here. With the near-boundary expansion in (4.19) we thus find

$$
\left\langle\mathcal{O}_{\theta}\right\rangle=-2 c, \quad\left\langle\mathcal{O}_{\varsigma}\right\rangle=\frac{2}{3} i \lambda c .
$$

With the regularity relation in (4.11) we see that the one-point functions are completely fixed in terms of the source, as expected for the field theory on $\mathrm{S}^{4}$. This is to be contrasted to the $\mathrm{AdS}_{4}$ slicing discussed in section 3, where the one-point functions could be dialed independently. 


\subsection{Sphere partition function}

We now compute the contribution of the defect fields to the $\mathrm{S}^{4}$ partition function, $\mathcal{F}\left(\mathrm{S}^{4}\right)$, to quadratic order in the mass deformation. The partition function is given by $\mathcal{F}\left(\mathrm{S}^{4}\right)=$ $-\mathrm{S}_{\text {on-shell,ren }}$, where $S_{\text {on-shell,ren }}$ is the combined on-shell action for type IIB supergravity and the DBI action describing the D5 branes. In the probe limit the contribution from the D5 branes is given by

$$
\delta \mathcal{F}\left(\mathrm{S}^{4}\right)=-\mathrm{S}_{\mathrm{D} 5 \text {,ren }},
$$

where $S_{\mathrm{D} 5 \text {,ren }}$ is the renormalized on-shell D5-brane action. The task at hand therefore is to compute $S_{\mathrm{D} 5 \text {,ren }}$ to quadratic order in $m$.

We can straightforwardly compute the contribution at $\mathcal{O}\left(m^{0}\right)$, which is well defined since there are no finite counterterms that could be added on the holographic side. This proceeds by directly integrating (4.13) and taking the finite part — the divergences are cancelled by the appropriate holographic counterterms without affecting the finite part which yields

$$
\left.S_{\mathrm{D} 5, \mathrm{ren}}\right|_{m=0}=\frac{4}{3} T_{5} V_{\mathrm{S}^{1}} V_{\mathrm{S}^{3}} .
$$

The term quadratic in $m$ can be conveniently computed using

$$
\begin{aligned}
\frac{\partial S_{\mathrm{D} 5}}{\partial m}=\kappa^{2}\left[\frac{\delta S_{\mathrm{D} 5}^{(2)}}{\delta \theta_{0}^{(1)}} \frac{\delta \theta_{0}^{(1)}}{\delta m}+\frac{\delta S_{\mathrm{D} 5}^{(2)}}{\delta \varsigma_{1}^{(1)}} \frac{\delta \varsigma_{1}^{(1)}}{\delta m}\right] & =T_{5} V_{\mathrm{S}^{1}} V_{\mathrm{S}^{3}}\left[\left\langle\mathcal{O}_{\theta}\right\rangle+3 i \lambda\left\langle\mathcal{O}_{\varsigma}\right\rangle\right] \\
& =-4 c T_{5} V_{\mathrm{S}^{1}} V_{\mathrm{S}^{3}}
\end{aligned}
$$

where (4.22) and (4.23) were used for the second and third equalities. We thus find, with the regularity relation $(4.11)$,

$$
S_{\mathrm{D} 5, \mathrm{ren}}=T_{5} V_{\mathrm{S}^{1}} V_{\mathrm{S}^{3}}\left[\frac{4}{3}+2 \kappa^{2} m^{2}+\mathcal{O}\left(\kappa^{3} m^{3}\right)\right] .
$$

To identify the parameters on the holographic side with those on the field theory side, we first introduce $T_{0}$ defined by $T_{\mathrm{D} 5} V_{\mathrm{S}^{2}}=T_{0}$, in analogy to the D3/D7 case in [6]. We then use $T_{0}=N_{f} N_{c} \sqrt{\lambda} /\left(2 \pi^{3}\right)$ from the table above eq. (4.18) in [29]. This yields

$$
T_{\mathrm{D} 5}=\frac{\mu}{4 \pi^{3}} N_{f} N_{c}, \quad \mu=\frac{\sqrt{\lambda}}{2 \pi} .
$$

With $V_{\mathrm{S}^{1}}=2 \pi$ and $V_{\mathrm{S}^{3}}=2 \pi^{2}$, we thus have $T_{\mathrm{D} 5} V_{\mathrm{S}^{1}} V_{\mathrm{S}^{3}}=\mu N_{f} N_{c}$. Finally, we note that the mass of the fundamental fields on the field theory side is given in terms of the leading term in the near-boundary expansion of the slipping mode by $M=\kappa m \mu$ [30]. Consequently, the final result for the defect contribution to the partition function reads

$$
\delta \mathcal{F}\left(\mathrm{S}^{4}\right)=-\mu \mathrm{N}_{\mathrm{f}} \mathrm{N}_{\mathrm{c}}\left[\frac{4}{3}+\frac{2 \mathrm{M}^{2}}{\mu^{2}}+\ldots\right]
$$

where the dots denote subleading terms in the mass and strong coupling expansions. 


\section{$5 \quad \mathrm{~S}^{4}$ partition function from supersymmetric localization}

In this section we switch to the field theory side and compute the contribution of the defect fields to the partition function on $\mathrm{S}^{4}$ using supersymmetric localization. We will start by discussing the matrix model that arises from the localization procedure and then evaluate the partition function in the quenched approximation where the number of defect fields is small compared to the rank of the gauge group in the background $\mathcal{N}=4 \mathrm{SYM}$ theory. This result can then be compared to the holographic computation of the same quantity in the previous section.

\subsection{The matrix model}

To fix notation we start with a brief review of supersymmetric localization. For a gauge theory described by a partition function $\mathcal{Z}$, one starts with the identification of a linear combination of a supercharge generating a supersymmetry that closes off shell and a BRST charge, $\mathcal{Q} \equiv Q_{\mathrm{SUSY}}+Q_{\mathrm{BRST}}$. The action is then deformed by a term $\mathcal{Q} V$ with appropriately chosen $V$ and this leaves the theory unchanged. That is, instead of the original partition one evaluates

$$
\mathcal{Z}(t)=\int D X e^{-S[X]-t \mathcal{Q} V[X]} .
$$

The original form of the partition function is recovered for $t=0$. But since $\partial_{t} \mathcal{Z}(t)=0$, we can equivalently evaluate it for $t \rightarrow \infty$, where the integral localizes to the saddle points of $\mathcal{Q} V$.

For $\mathrm{SU}\left(N_{c}\right) \mathcal{N}=4 \mathrm{SYM}$ with conveniently chosen $V$, the saddles are parametrized by a single adjoint matrix, and the resulting expression for the partition function on $\mathrm{S}^{4}$ is [1]

$$
\mathcal{Z}=\int d a^{N_{c}-1} \prod_{i<j} a_{[i j]}^{2} e^{S_{0}}, \quad S_{0}=-\frac{8 \pi^{2}}{\lambda} N_{c} \sum_{i} a_{i}^{2},
$$

where $a_{[i j]}=a_{i}-a_{j}$ labels the roots of $\mathfrak{s u}\left(N_{c}\right)$ and $a_{i}$ labels the weights. Notably, the contributions of the one-loop fluctuations of the $\mathcal{N}=4$ SYM fields around the saddles of $\mathcal{Q} V$ cancel out and do not appear in this expression. For $\mathcal{N}=4 \mathrm{SYM}$ coupled to fundamental fields, the saddles of $\mathcal{Q} V$ are still parametrized by a single adjoint matrix [1], and the only change in the partition function is the contribution of the 1-loop fluctuations of the fundamental fields around the saddles. We used this result in [6] to compare the holographic computation of the partition function to a field theory computation.

This story could potentially change when deforming $\mathcal{N}=4 \mathrm{SU}\left(N_{c}\right)$ SYM theory on an $\mathrm{S}^{4}$ by adding a defect matter sector. We can no longer rely on the matrix model constructed out of the components listed in [1]. Generally, we would start from scratch to derive the new localized theory and compute the one-loop determinants. However, inspired by the way the computation proceeds for gauge theories coupled to spacetime-filling matter fields, we will take a more pragmatic route here as follows. The form of the one-loop determinant for $N_{f}$ (massive) fundamental hypermultiplets on an $\mathrm{S}^{3}$, coupled to an intrinsically 3-dimensional superconformal Chern-Simons theories has been given in [33] and reads

$$
\prod_{i} \operatorname{sech}^{N_{f}}\left(\pi\left(a_{i}+M\right)\right),
$$


where $M$ is the mass parameter. We will assume that, with appropriately chosen $V$, the path integral for the defect theory we are considering once again localizes to saddle points parametrized by the single adjoint matrix setting the constant value for one of the scalar fields in the $\mathcal{N}=4 \mathrm{SYM}$ multiplet. The only contribution of the defect fields would then again be their one-loop fluctuations, and the matrix model we have to solve is

$$
\mathcal{Z}_{\text {defect }}=\int d a^{N_{c}-1} \prod_{i<j} a_{[i j]}^{2} \frac{1}{\prod_{i} \cosh ^{N_{f}}\left(\pi\left(a_{i}+M\right)\right)} e^{S_{0}} .
$$

This procedure for constructing the matrix model may be further motivated by the observation that the localizing supercharge $\mathcal{Q}$ and the cohomological deformation $\mathcal{Q} V$ used for the three-dimensional theories in [33] are the same as those used for the four-dimensional theory in [1]. This was pointed out in appendix B of [34].

\section{2 $S^{4}$ partition function}

To evaluate the partition function (5.4) in the limit of strong coupling and large $N_{c}$, with $N_{f} \ll N_{c}$, we first rearrange the integrand such that all factors appear in the exponent. This yields, with $\zeta=N_{f} / N_{c}$,

$$
\mathcal{Z}_{\text {defect }}=\int d a^{N_{c}-1} e^{S}, \quad S=S_{0}+\sum_{i<j} \log a_{[i j]}^{2}-N_{c} \zeta \sum_{i} \log \left(\cosh \left(\pi\left(a_{i}+M\right)\right)\right.
$$

The first two terms in $S$ are $\mathcal{O}\left(N_{c}^{2}\right)$, while the last term is $\mathcal{O}\left(N_{f} N_{c}\right)$ and provides the contribution of the defect fields. In the quenched approximation with $N_{f} \ll N_{c}$ the computation of the partition function can be organized in an expansion in $\zeta$, by using that we are close to the solution for the matrix model for pure $\mathcal{N}=4 \mathrm{SYM}$. The expansion of the "on-shell" action reads

$$
\frac{S}{N_{c}^{2}}=\left.\tilde{S}_{0}\right|_{\rho_{0}}+\zeta\left(\left.S_{1}\right|_{\rho_{0}}+\left.\delta \tilde{S}_{0}\right|_{\rho_{0}}\right)+\mathcal{O}\left(\zeta^{2}\right)
$$

where we have defined $\tilde{S}_{0}$ by $N_{c}^{2} \tilde{S}_{0}=S_{0}+\sum_{i<j} \log a_{[i j]}^{2}$ and $S_{1}$ is the contribution of the defect fields. We have denoted by $\rho_{0}$ the solution to the Gaussian matrix model corresponding to pure $\mathcal{N}=4 \mathrm{SYM}$ in the continuum limit, which is the Wigner semicircle distribution

$$
\rho_{0}(x)=\frac{2}{\pi \mu^{2}} \sqrt{\mu^{2}-x^{2}}, \quad \mu=\frac{\sqrt{\lambda}}{2 \pi} .
$$

The great simplification in the quenched approximation is that, since $\rho_{0}$ extremizes $\tilde{S}_{0}$, $\left.\delta \tilde{S}_{0}\right|_{\rho_{0}}=0$ and we only need $\left.S_{1}\right|_{\rho_{0}}$ to compute the contribution of the defect fields to the partition function at leading order. The contribution of the defect fields thus evaluates to

$$
\delta \mathcal{F}=\left.N_{c}^{2} \zeta S_{1}\right|_{\rho_{0}},\left.\quad S_{1}\right|_{\rho_{0}}=-\int_{-\mu}^{\mu} d x \rho_{0}(x) \log \cosh (\pi(x+M)) .
$$

With a simple change of variables, setting $x=\mu y$, and defining $m$ by $M=\mu m$, we can rewrite this as

$$
\delta \mathcal{F}=-\frac{2 N_{c}^{2} \zeta}{\pi} \int_{-1}^{1} d y \sqrt{1-y^{2}} \log \cosh (\mu \pi(y+m))
$$


In analogy to the holographic computation we will evaluate this integral in an expansion for small $m$. The $m=0$ contribution can be evaluated straightforwardly, yielding

$$
\begin{aligned}
\left.\delta \mathcal{F}\right|_{m=0} & =-\frac{4 N_{c}^{2} \zeta}{\pi} \int_{0}^{1} d y \sqrt{1-y^{2}} \log \cosh (\pi \mu y) \\
& \approx-\frac{4 \mu N_{c}^{2} \zeta}{\pi} \int_{0}^{1} d y \sqrt{1-y^{2}} \pi \mu y=-\frac{4}{3} \mu N_{c}^{2} \zeta,
\end{aligned}
$$

where we used $\mu \gg 1$ and the large argument expansion for the cosh in the second line. This is justified except for a region with width of order $1 / \mu$ around the origin. But since the integrand is smooth there the contribution from this region is subleading in $\mu$.

To compute the first term in the Taylor expansion of $\delta F$ around $m=0$, we evaluate

$$
\left.\frac{d \delta \mathcal{F}}{d m}\right|_{m=0}=-2 \mu N_{c}^{2} \zeta \int_{-1}^{1} d y \sqrt{1-y^{2}} \tanh (\pi \mu y) .
$$

The integrand is odd under $y \rightarrow-y$ and the integration domain symmetric, so $d \delta \mathcal{F} /\left.d m\right|_{m=0}$ vanishes. The next term in the expansion can be computed from

$$
\left.\frac{d^{2} \delta \mathcal{F}}{d m^{2}}\right|_{m=0}=-2 \pi \mu^{2} N_{c}^{2} \zeta \int_{-1}^{1} d y \sqrt{1-y^{2}} \operatorname{sech}^{2}(\pi \mu y) .
$$

For large $\mu$ the $\operatorname{sech}^{2}(\pi \mu y)$ factor decays exponentially away from $y=0$, so the dominant contribution comes from an exponentially small region around $y=0$. At small $y$, we can expand $\sqrt{1-y^{2}} \approx 1-y^{2} / 2$, and since the contributions from regions where $y$ is $\mathcal{O}(1)$ are exponentially suppressed, we can use this expansion for the entire domain of integration. As a result, to leading order in large $\mu$,

$$
\left.\frac{d^{2} \delta \mathcal{F}}{d m^{2}}\right|_{m=0}=-2 \pi \mu^{2} N_{c}^{2} \zeta \int_{-1}^{1} d y \operatorname{sech}^{2}(\pi \mu y)=-4 \mu N_{c}^{2} \zeta
$$

The explicit form of the contribution of the defect fields to the partition function, to quadratic order in $m$, therefore reads

$$
\delta \mathcal{F}=\mu N_{f} N_{c}\left(-\frac{4}{3}-2 m^{2}+\ldots\right)=-\mu N_{f} N_{c}\left(\frac{4}{3}+\frac{2 M^{2}}{\mu^{2}}+\ldots\right) .
$$

This matches precisely the result of the holographic computation in (4.29). It validates not only the holographic computation but also lends support to the construction of the matrix model outlined in the previous section and resulting in (5.4). Extending the matrix model computation to higher orders in $m$ is straightforward, both in principle and in practice, while the holographic computation gets considerably more involved at higher orders in $m$. But already the computation thus far provides a strong check and we certainly expect the higher orders to agree as well.

\section{Acknowledgments}

We are very happy to thank Andreas Karch for many useful discussions. The work of BR is supported, in part, by the U.S. Department of Energy under Grant No. DE-SC0011637. The work of CFU is supported, in part, by the U.S. Department of Energy under Grant No. DE-SC0011637 and by the National Science Foundation under grant PHY-16-19926. 


\section{A Details on non-linear $\kappa$-symmetry}

In this appendix we provide the details for the derivation of the non-linear $\kappa$-symmetry conditions discussed in section 2.4. The basic idea will be to isolate the $\mathrm{S}^{5}$ and $\mathrm{AdS}_{5} \mathrm{Clifford}$ algebra structures and use the massive projector as well as the chirality constraint on the constant spinor $\epsilon_{0}$ to reduce to a minimal number of $\mathrm{AdS}_{5}$ Clifford algebra structures, which then have to vanish independently. The starting point is the $\kappa$-symmetry condition as spelled out in (2.57), which we repeat for convenience

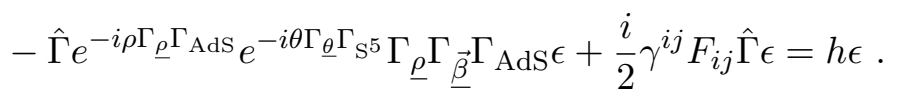

To evaluate $\hat{\Gamma}$ explicitly, we start from (2.17), (2.18) and decompose

$$
\gamma_{r \beta_{1} \beta_{2}}=\mathcal{A}^{\mathbb{1}}+\mathcal{A}^{\rho} \Gamma_{\rho}+\mathcal{A}^{r} \Gamma_{r}+A^{\rho r} \Gamma_{\rho r},
$$

where, with $\tau^{\mathfrak{m}}=\varepsilon^{\mathfrak{m} \mathfrak{m} r}(d \rho)_{\mathfrak{n}}(d \theta)_{\mathfrak{r}}$ and $\varepsilon^{r \beta_{1} \beta_{2}}=1$, the $S^{5}$ Clifford algebra structures are given by

$$
\begin{aligned}
\mathcal{A}^{\mathbb{1}} & =(d \theta)_{r} \Gamma_{\theta} \Gamma_{\vec{\beta}}, & \mathcal{A}^{\rho} & =(d \rho)_{r} \Gamma_{\vec{\beta}}-\tau^{\beta_{i}} \Gamma_{\beta_{i}} \Gamma_{\theta}, \\
A^{r} & =\Gamma_{\vec{\beta}}-\varepsilon^{r \mathfrak{m n}}(d \theta)_{\mathfrak{m}} \Gamma_{\mathfrak{n}} \Gamma_{\theta}, & A^{\rho r} & =\varepsilon^{r \mathfrak{m n}}(d \rho)_{\mathfrak{m}} \Gamma_{\mathfrak{n}}-\tau^{r} \Gamma_{\theta} .
\end{aligned}
$$

With (A.2) and $\Gamma_{11} \epsilon=\epsilon$ we then find

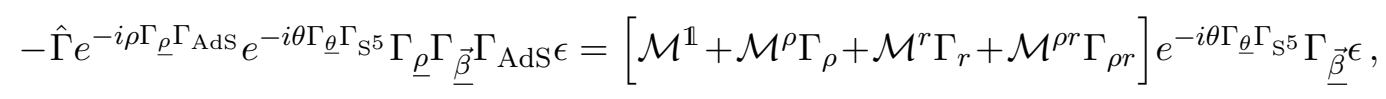

where

$$
\begin{aligned}
\mathcal{M}^{\mathbb{1}} & =-\cosh ^{2} \rho\left(\mathcal{A}^{r}+i \tanh \rho A^{\rho r} \Gamma_{\mathrm{S}^{5}}\right), & \mathcal{M}^{\rho} & =-\cosh ^{2} \rho\left(A^{\rho r}-i \tanh \rho \mathcal{A}^{r} \Gamma_{\mathrm{S}^{5}}\right), \\
\mathcal{M}^{r} & =-\mathcal{A}^{\mathbb{1}}-i \tanh \rho \mathcal{A}^{\rho} \Gamma_{\mathrm{S}^{5}}, & \mathcal{M}^{\rho r} & =-\mathcal{A}^{\rho}+i \tanh \rho \mathcal{A}^{\mathbb{1}} \Gamma_{\mathrm{S}^{5}} .
\end{aligned}
$$

Likewise, we decompose the $\gamma \cdot F$ term into $\mathrm{AdS}_{5}$ and $\mathrm{S}^{5}$ Clifford algebra structures and find

$$
\frac{1}{2} \gamma^{i j} F_{i j}=\mathcal{B}^{\mathbb{1}}+\mathcal{B}^{\rho} \Gamma_{\rho}+\mathcal{B}^{r} \Gamma_{r}+B^{\rho r} \Gamma_{\rho r},
$$

with the $\mathrm{S}^{5}$ Clifford algebra structures

$$
\begin{array}{ll}
B^{\mathbb{1}}=(d \theta)_{r} F^{r \beta_{i}} \Gamma_{\theta} \Gamma_{\beta_{i}}+F^{\beta_{1} \beta_{2}}\left(\Gamma_{\vec{\beta}}+\Gamma_{\theta} \varepsilon^{r \mathfrak{m n}}(d \theta)_{\mathfrak{m}} \Gamma_{\mathfrak{n}}\right), & B^{r}=-F^{r \beta_{i}}\left(\Gamma_{\beta_{i}}+(d \theta)_{\beta_{i}} \Gamma_{\theta}\right), \\
B^{\rho}=F^{\mathfrak{m} \mathfrak{n}}(d \theta)_{\mathfrak{m}}(d \rho)_{\mathfrak{n}} \Gamma_{\theta}+F^{\beta_{i} \mathfrak{m}}(d \rho)_{\mathfrak{m}} \Gamma_{\beta_{i}}, & B^{\rho r}=-F^{r \beta_{i}}(d \rho)_{\beta_{i}} \mathbb{\mathbb { 1 }}
\end{array}
$$

For the combination appearing in (A.1) this yields

$$
\frac{i}{2} \gamma^{i j} F_{i j} \hat{\Gamma} \epsilon=-i\left[\mathcal{E}^{\mathbb{1}}+\mathcal{E}^{\rho} \Gamma_{\rho}+\mathcal{E}^{r} \Gamma_{r}+\mathcal{E}^{\rho e} \Gamma_{\rho r}\right] \Gamma_{\mathrm{S}^{5}} \epsilon
$$


where

$$
\begin{aligned}
\mathcal{E}^{\mathbb{1}} & =-\cosh \rho\left(\mathcal{B}^{\rho r} \mathcal{A}^{\mathbb{1}}+\mathcal{B}^{\mathbb{1}} \mathcal{A}^{\rho r}+\mathcal{B}^{\rho} \mathcal{A}^{r}-\mathcal{B}^{r} \mathcal{A}^{\rho}\right), \\
\mathcal{E}^{\rho} & =-\cosh \rho\left(\mathcal{B}^{r} \mathcal{A}^{\mathbb{1}}-\mathcal{B}^{\mathbb{1}} \mathcal{A}^{r}+\mathcal{B}^{\rho} \mathcal{A}^{\rho r}+\mathcal{B}^{\rho r} \mathcal{A}^{\rho}\right), \\
\mathcal{E}^{r} & =\operatorname{sech} \rho\left(\mathcal{B}^{\rho} \mathcal{A}^{\mathbb{1}}-\mathcal{B}^{\mathbb{1}} \mathcal{A}^{\rho}\right)-\cosh \rho\left(\mathcal{B}^{r} \mathcal{A}^{\rho r}+\mathcal{B}^{\rho r} \mathcal{A}^{r}\right), \\
\mathcal{E}^{\rho r} & =\operatorname{sech} \rho\left(\mathcal{B}^{\mathbb{1}} \mathcal{A}^{\mathbb{1}}+\mathcal{B}^{\rho} \mathcal{A}^{\rho}\right)+\cosh \rho\left(\mathcal{B}^{r} \mathcal{A}^{r}-\mathcal{B}^{\rho r} \mathcal{A}^{\rho r}\right) .
\end{aligned}
$$

We can then assemble the full $\kappa$-symmetry condition, which becomes

$$
\left[\mathcal{N}^{\mathbb{1}}+\mathcal{N}^{\rho} \Gamma_{\rho}+\mathcal{N}^{r} \Gamma_{r}+\mathcal{N}^{\rho r} \Gamma_{\rho r}\right] \epsilon=h \epsilon
$$

where the $\mathrm{S}^{5}$ Clifford algebra structures are given by

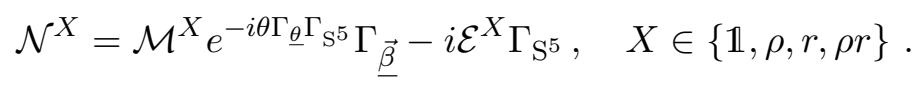

We can now use the massive projector (2.52) to reduce the number of Clifford algebra structures as follows. Multiplying eq. (A.10) by $R_{\mathrm{AdS}}^{-1} R_{\mathrm{S}^{5}}^{-1}$ yields

$$
\left(\mathcal{R}_{S}\left[\mathcal{N}^{\mathbb{1}}\right]+\mathcal{R}_{S}\left[\mathcal{N}^{\rho}\right] \mathcal{R}_{A}\left[\Gamma_{\rho}\right]+\mathcal{R}_{S}\left[\mathcal{N}^{r}\right] \mathcal{R}_{A}\left[\Gamma_{r}\right]+\mathcal{R}_{S}\left[\mathcal{N}^{\rho r}\right] \mathcal{R}_{A}\left[\Gamma_{\rho r}\right]\right) \epsilon_{0}=h \epsilon_{0},
$$

where we use the definitions of $\mathcal{R}_{S}$ and $\mathcal{R}_{A}$ as given in (2.47). We now use the following identities

$$
\begin{aligned}
\mathcal{R}_{A}\left[\Gamma_{\underline{\rho}}\right] & =\cosh r \mathcal{K} \Gamma_{\underline{\rho}}+i \sinh r \Gamma_{\underline{\rho r}} \Gamma_{\text {AdS }}, \\
\mathcal{R}_{A}\left[\Gamma_{\underline{r}}\right] & =\mathcal{K}\left(\cosh \rho \Gamma_{\underline{r}}-\sinh \rho \sinh r \Gamma_{\underline{\rho}}\right)-i \sinh \rho \cosh r \Gamma_{\underline{\rho r}} \Gamma_{\mathrm{AdS}}, \\
\mathcal{R}_{A}\left[\Gamma_{\underline{\rho} r}\right] & =-i \mathcal{K}\left(\sinh \rho \Gamma_{\underline{r}}-\cosh \rho \sinh r \Gamma_{\underline{\rho}}\right) \Gamma_{\mathrm{AdS}}+\cosh \rho \cosh r \Gamma_{\underline{\rho r}},
\end{aligned}
$$

where the matrix $\mathcal{K}$ encodes the entire dependence on the $\mathrm{AdS}_{3}$ directions and is given by

$$
\mathcal{K}=\left[e^{-x}+e^{x}\left(y^{2}-t^{2}\right)\right] P_{x+}+e^{x} P_{x-}-i e^{x}\left(t \Gamma_{\underline{t}}+y \Gamma_{\underline{y}}\right) \Gamma_{\text {AdS }} .
$$

The projectors $P_{x \pm}$ were defined above (2.8). We now use that these structures act on $\epsilon_{0}$, which satisfies $\Gamma_{11} \epsilon_{0}=\epsilon_{0}$ and the massive projection condition (2.52). So we have $\Gamma_{\text {AdS }} \epsilon_{0}=-\Gamma_{\mathrm{S}^{5}} \epsilon_{0}$ and $\Gamma_{\underline{\rho}} \epsilon_{0}=-\hat{\Gamma}_{p} \epsilon_{0}$, with $\hat{\Gamma}_{p}=\frac{\lambda}{|c|} \Gamma_{p}$ such that $\hat{\Gamma}_{p}^{2}=-\mathbb{1}$. This yields

$$
\begin{aligned}
\mathcal{R}_{A}\left[\Gamma_{\underline{\rho}}\right] \epsilon_{0} & =\cosh r \mathcal{K} \Gamma_{\underline{\rho}} \epsilon_{0}+i \sinh r \hat{\Gamma}_{p} \Gamma_{\mathrm{S}^{5}} \epsilon_{0}, \\
\mathcal{R}_{A}\left[\Gamma_{\underline{r}}\right] \epsilon_{0} & =-\left(\cosh \rho \hat{\Gamma}_{p}+\sinh \rho \sinh r \mathbb{1}\right) \mathcal{K} \Gamma_{\underline{\rho}} \epsilon_{0}-i \sinh \rho \cosh r \hat{\Gamma}_{p} \Gamma_{\mathrm{S}^{5}} \epsilon_{0}, \\
\mathcal{R}_{A}\left[\Gamma_{\underline{\rho} r}\right] \epsilon_{0} & =i\left(\sinh \rho \hat{\Gamma}_{p}+\cosh \rho \sinh r \mathbb{1}\right) \Gamma_{\mathrm{S}^{5}} \mathcal{K} \Gamma_{\underline{\rho}} \epsilon_{0}-\cosh \rho \cosh r \hat{\Gamma}_{p} \epsilon_{0} .
\end{aligned}
$$

We also used that $\hat{\Gamma}_{p}$ has an even number of $\mathrm{S}^{5} \Gamma$-matrices and commutes with AdS $\Gamma$ matrices, and that $\mathcal{K}$ commutes with $\mathrm{S}^{5} \Gamma$-matrices. With these identities, eq. (A.12) becomes

$$
\mathcal{Q}_{\mathcal{K}} \mathcal{K} \Gamma_{\underline{\rho}} \epsilon_{0}+\mathcal{Q}_{\mathbb{1}} \epsilon_{0}=h \epsilon_{0},
$$


where

$$
\begin{aligned}
\mathcal{Q}_{\mathcal{K}}= & \cosh r \mathcal{R}_{S}\left[\mathcal{N}^{\rho}\right]-\cosh ^{2} \rho \mathcal{R}_{S}\left[\mathcal{N}^{r}\right]\left(\hat{\Gamma}_{p}+\tanh \rho \sinh r \mathbb{1}\right) \\
& +i \cosh ^{2} \rho \mathcal{R}_{S}\left[\mathcal{N}^{\rho r}\right]\left(\tanh \rho \hat{\Gamma}_{p}+\sinh r \mathbb{1}\right) \Gamma_{\mathrm{S}^{5}}, \\
\mathcal{Q}_{\mathbb{1}}= & \mathcal{R}_{S}\left[\mathcal{N}^{\mathbb{1}}\right]+i \sinh r \mathcal{R}_{S}\left[\mathcal{N}^{\rho}\right] \hat{\Gamma}_{p} \Gamma_{\mathrm{S}^{5}}-i \sinh \rho \cosh \rho \cosh r \mathcal{R}_{S}\left[\mathcal{N}^{r}\right] \hat{\Gamma}_{p} \Gamma_{S^{5}} \\
& -\cosh ^{2} \rho \cosh r \mathcal{R}_{S}\left[\mathcal{N}^{\rho r}\right] \hat{\Gamma}_{p} .
\end{aligned}
$$

We have implemented all the projectors and chirality conditions we have at our disposal to reduce the AdS Clifford algebra structures to just one, and we can hence now formulate the conditions for $\kappa$-symmetry as two operator equations which have to be satisfied simultaneously. This leads to the conditions in eq. (2.60).

Open Access. This article is distributed under the terms of the Creative Commons Attribution License (CC-BY 4.0), which permits any use, distribution and reproduction in any medium, provided the original author(s) and source are credited.

\section{References}

[1] V. Pestun, Localization of gauge theory on a four-sphere and supersymmetric Wilson loops, Commun. Math. Phys. 313 (2012) 71 [arXiv:0712.2824] [INSPIRE].

[2] N. Hama, K. Hosomichi and S. Lee, SUSY Gauge Theories on Squashed Three-Spheres, JHEP 05 (2011) 014 [arXiv:1102.4716] [INSPIRE].

[3] N. Bobev, H. Elvang, D.Z. Freedman and S.S. Pufu, Holography for $N=2^{*}$ on $S^{4}$, JHEP 07 (2014) 001 [arXiv: 1311.1508] [INSPIRE].

[4] M. Mariño, Lectures on localization and matrix models in supersymmetric Chern-Simons-matter theories, J. Phys. A 44 (2011) 463001 [arXiv:1104.0783] [InSPIRE].

[5] L.F. Alday, M. Fluder, P. Richmond and J. Sparks, Gravity Dual of Supersymmetric Gauge Theories on a Squashed Five-Sphere, Phys. Rev. Lett. 113 (2014) 141601 [arXiv:1404.1925] [INSPIRE].

[6] A. Karch, B. Robinson and C.F. Uhlemann, Precision Test of Gauge-Gravity Duality with Flavor, Phys. Rev. Lett. 115 (2015) 261601 [arXiv:1509.00013] [INSPIRE].

[7] J.R. David, E. Gava, R.K. Gupta and K. Narain, Localization on $A d S_{2} \times S^{1}$, JHEP 03 (2017) 050 [arXiv : 1609. 07443] [INSPIRE].

[8] F. Bonetti and L. Rastelli, Supersymmetric Localization in $A d S_{5}$ and the Protected Chiral Algebra, arXiv:1612.06514 [INSPIRE].

[9] P. Breitenlohner and D.Z. Freedman, Positive Energy in anti-de Sitter Backgrounds and Gauged Extended Supergravity, Phys. Lett. B 115 (1982) 197 [InSPIRE].

[10] P. Breitenlohner and D.Z. Freedman, Stability in Gauged Extended Supergravity, Annals Phys. 144 (1982) 249 [INSPIRE].

[11] O. Aharony, D. Marolf and M. Rangamani, Conformal field theories in anti-de Sitter space, JHEP 02 (2011) 041 [arXiv: 1011.6144] [INSPIRE].

[12] E. Gava, K.S. Narain, M.N. Muteeb and V.I. Giraldo-Rivera, $N=2$ gauge theories on the hemisphere $H S^{4}$, Nucl. Phys. B 920 (2017) 256 [arXiv: 1611.04804] [INSPIRE]. 
[13] E. Gerchkovitz, J. Gomis and Z. Komargodski, Sphere Partition Functions and the Zamolodchikov Metric, JHEP 11 (2014) 001 [arXiv:1405.7271] [INSPIRE].

[14] A. Karch, B. Robinson and C.F. Uhlemann, Supersymmetric D3/D7 for holographic flavors on curved space, JHEP 11 (2015) 112 [arXiv:1508.06996] [INSPIRE].

[15] A. Karch and E. Katz, Adding flavor to AdS/CFT, JHEP 06 (2002) 043 [hep-th/0205236] [INSPIRE].

[16] O. Aharony, O. DeWolfe, D.Z. Freedman and A. Karch, Defect conformal field theory and locally localized gravity, JHEP 07 (2003) 030 [hep-th/0303249] [INSPIRE].

[17] O. DeWolfe, D.Z. Freedman and H. Ooguri, Holography and defect conformal field theories, Phys. Rev. D 66 (2002) 025009 [hep-th/0111135] [INSPIRE].

[18] I. Buhl-Mortensen, M. de Leeuw, A.C. Ipsen, C. Kristjansen and M. Wilhelm, A Quantum Check of AdS/dCFT, JHEP 01 (2017) 098 [arXiv:1611.04603] [INSPIRE].

[19] I. Buhl-Mortensen, M. de Leeuw, A.C. Ipsen, C. Kristjansen and M. Wilhelm, One-loop one-point functions in gauge-gravity dualities with defects, Phys. Rev. Lett. 117 (2016) 231603 [arXiv: 1606.01886] [INSPIRE].

[20] M. de Leeuw, C. Kristjansen and K. Zarembo, One-point Functions in Defect CFT and Integrability, JHEP 08 (2015) 098 [arXiv: 1506.06958] [INSPIRE].

[21] M. de Leeuw, A.C. Ipsen, C. Kristjansen, K.E. Vardinghus and M. Wilhelm, Two-point functions in AdS/dCFT and the boundary conformal bootstrap equations, JHEP 08 (2017) 020 [arXiv: 1705. 03898] [inSPIRE].

[22] E. Widen, Two-point functions of $\mathrm{SU}(2)$-subsector and length-two operators in dCFT, Phys. Lett. B 773 (2017) 435 [arXiv:1705.08679] [INSPIRE].

[23] M. Preti, D. Trancanelli and E. Vescovi, Quark-antiquark potential in defect conformal field theory, JHEP 10 (2017) 079 [arXiv: 1708.04884] [INSPIRE].

[24] L. Rastelli and X. Zhou, The Mellin Formalism for Boundary CFT [arXiv: 1705.05362] [INSPIRE].

[25] G. Festuccia and N. Seiberg, Rigid Supersymmetric Theories in Curved Superspace, JHEP 06 (2011) 114 [arXiv: 1105.0689] [INSPIRE].

[26] E. Bergshoeff and P.K. Townsend, Super D-branes, Nucl. Phys. B 490 (1997) 145 [hep-th/9611173] [INSPIRE].

[27] M. Cederwall, A. von Gussich, B.E.W. Nilsson and A. Westerberg, The Dirichlet super three-brane in ten-dimensional type IIB supergravity, Nucl. Phys. B 490 (1997) 163 [hep-th/9610148] [INSPIRE].

[28] M. Cederwall, A. von Gussich, B.E.W. Nilsson, P. Sundell and A. Westerberg, The Dirichlet super p-branes in ten-dimensional type IIA and IIB supergravity, Nucl. Phys. B 490 (1997) 179 [hep-th/9611159] [INSPIRE].

[29] H.-C. Chang and A. Karch, Entanglement Entropy for Probe Branes, JHEP 01 (2014) 180 [arXiv:1307.5325] [INSPIRE].

[30] M. Kruczenski, D. Mateos, R.C. Myers and D.J. Winters, Meson spectroscopy in AdS/CFT with flavor, JHEP 07 (2003) 049 [hep-th/0304032] [INSPIRE].

[31] M.T. Grisaru, R.C. Myers and O. Tafjord, SUSY and goliath, JHEP 08 (2000) 040 [hep-th/0008015] [INSPIRE]. 
[32] D.Z. Freedman, K. Pilch, S.S. Pufu and N.P. Warner, Boundary Terms and Three-Point Functions: An AdS/CFT Puzzle Resolved, JHEP 06 (2017) 053 [arXiv:1611.01888] [INSPIRE].

[33] A. Kapustin, B. Willett and I. Yaakov, Exact Results for Wilson Loops in Superconformal Chern-Simons Theories with Matter, JHEP 03 (2010) 089 [arXiv:0909.4559] [InSPIRE].

[34] N. Drukker, D. Gaiotto and J. Gomis, The Virtue of Defects in $4 D$ Gauge Theories and $2 D$ CFTs, JHEP 06 (2011) 025 [arXiv: 1003.1112] [INSPIRE]. 